\title{
Aberrant cortical development is driven by impaired cell cycle and translational control in a $D D X 3 X$ syndrome model
}

Mariah L. Hoye ${ }^{1}$, Lorenzo Calviello ${ }^{2,3}$, Abigail J. Poff ${ }^{1}$, Nna-Emeka Ejimogu ${ }^{1}$, Carly R. Newman ${ }^{1}$, Jianhong Ou ${ }^{4,5}$, Stephen N. Floor ${ }^{6,7}$, Debra L. Silver ${ }^{1,4,5,8,9}$ *

${ }^{1}$ Department of Molecular Genetics and Microbiology, Duke University Medical Center, Durham, NC 27710, USA

${ }^{2}$ Centre for Functional Genomics, Human Technopole, Milan, 20157, Italy

${ }^{3}$ Centre for Computational Biology, Human Technopole, Milan, 20157, Italy

${ }^{4}$ Department of Cell Biology, Duke University Medical Center, Durham, NC 27710, USA

${ }^{5}$ Duke Regeneration Center, Duke University Medical Center, Durham, NC 27710, USA

${ }^{6}$ Department of Cell and Tissue Biology, UCSF, San Francisco, CA 94158, USA

${ }^{7}$ Helen Diller Family Comprehensive Cancer Center, San Francisco, CA, 94143, USA

${ }^{8}$ Department of Neurobiology, Duke University Medical Center, Durham, NC 27710, USA

${ }^{9}$ Duke Institute for Brain Sciences, Duke University Medical Center, Durham, NC 27710, USA

*corresponding author: debra.silver@duke.edu

Keywords: cortical development, RNA helicase, translation, DDX3X syndrome, neurogenesis, radial glia 


\begin{abstract}
Mutations in the RNA helicase, $D D X 3 X$, are a leading cause of Intellectual Disability and present as $D D X 3 X$ syndrome, a neurodevelopmental disorder associated with cortical malformations and autism. Yet the cellular and molecular mechanisms by which $D D X 3 X$ controls cortical development are largely unknown. Here, using a mouse model of $D d x 3 x$ loss-of-function we demonstrate that DDX $3 \mathrm{X}$ directs translational and cell cycle control of neural progenitors, which underlies precise corticogenesis. First, we show brain development is highly sensitive to $D d x 3 x$ dosage; Complete $D d x 3 x$ loss from neural progenitors causes microcephaly in females, whereas hemizygous males and heterozygous females show reduced neurogenesis without marked microcephaly. In addition, $D d x 3 x$ loss is sexually dimorphic, as its paralog, $D d x 3 y$, compensates for $D d x 3 x$ in the developing male neocortex. Using live imaging of progenitors, we show that DDX3X promotes neuronal generation by regulating both cell cycle duration and neurogenic divisions. Finally, we use ribosome profiling in vivo to discover the repertoire of translated transcripts in neural progenitors, including those which are DDX3X-dependent and essential for neurogenesis. Our study reveals invaluable new insights into the etiology of $D D X 3 X$ syndrome, implicating dysregulated progenitor cell cycle dynamics and translation as pathogenic mechanisms.
\end{abstract}




\section{Introduction}

The cerebral cortex is critical for higher-order cognitive, motor, and sensory functioning. These processes rely upon embryonic development, when the proper number and types of neurons are generated (Kriegstein and Alvarez-Buylla 2009; Lodato and Arlotta 2015; Silver DL 2019). At the onset of cortical development, neuroepithelial cells undergo symmetric divisions to expand the progenitor pool before transitioning into radial glial cells (RGCs). In the ventricular zone (VZ), RGCs undergo symmetric self-renewing divisions or asymmetric divisions to produce either intermediate progenitors (IPs) or neurons. IPs, also referred to as basal progenitors, divide 1-2 times in the sub-ventricular zone (SVZ) before terminally differentiating into neurons. While mouse neurogenesis largely relies on RGCs and IPs, humans have a more expansive neurogenic basal progenitor population. Excitatory neurons are produced in an inside-out fashion; wherein deep layers (V/VI) are the earliest born, followed by production of superficial neurons (IV-II/III). Newborn neurons migrate to the cortical plate (CP) where they differentially project axons depending on their laminar position. Impairments in these steps of cortical development can cause neurodevelopmental disorders, including microcephaly, intellectual disability (ID), and autism spectrum disorder (ASD) (Willsey et al. 2013; Polioudakis et al. 2019; Willsey et al. 2021).

De novo mutations in the RNA helicase, $D D X 3 X$, are one of the leading causes of ID in females and underlie DDX3X syndrome (Snijders Blok et al. 2015; Wang et al. 2018; Beal et al. 2019; Scala et al. 2019; Lennox et al. 2020). DDX3X is X-linked, which likely explains the preponderance of female cases; although an increasing number of de novo and inherited $D D X 3 X$ mutations are found in males (Kellaris et al. 2018; Nicola et al. 2019). While DDX3X syndrome is characterized by ID, these individuals also commonly present with muscle tone and gait abnormalities, language deficits, abnormal brain MRIs (particularly white matter loss and corpus callosum defects), and many are diagnosed with ASD (Johnson-Kerner et al. 1993; Lennox et al. 2020; Tang et al. 2021). Indeed, DDX3X mutations have been identified in ASD cohorts, and 
$D D X 3 X$ is considered a high-confidence Autism gene (lossifov et al. 2014; RK et al. 2017; Takata et al. 2018; Ruzzo et al. 2019). DDX3X mutations have also been linked to cancer progression, including medulloblastoma (Jones et al. 2012; Pugh et al. 2012; Robinson et al. 2012), many of which overlap with ID-associated mutations. This oncogenic role is consistent with conserved functions of DDX3X in cell cycle progression (Li et al. 2014; Chen et al. 2016a; Kotov et al. 2016; Zhang and Li 2021). Notably, cell cycle duration is also strongly implicated in cortical development (Lange et al. 2009; Pilaz et al. 2009; Arai et al. 2011; Pilaz et al. 2016). Altogether, these clinical findings argue that $D D X 3 X$ mutations are deleterious and suggest that cell cycle defects may underlie their association with cancer and ID.

Remarkably, over 100 de novo mutations have been identified in DDX3X syndrome, equally composed of nonsense/frameshift and missense (Johnson-Kerner et al. 1993). The former class likely results in $D D X 3 X$ haploinsufficiency and/or hypomorphic loss-of-function (LoF) (Fig. 1A). Consistent with this, in female mice, $D d x 3 x$ germline haploinsufficiency impairs postnatal brain architecture and causes behavioral deficits phenocopying aspects of human DDX3X syndrome (Boitnott et al. 2021). In addition, using transient CRISPR approaches we previously showed that acute $D d x 3 x$ depletion in a subset of cells perturbed progenitor and neuron number (Lennox et al. 2020). While these studies highlight the requirement of $D d x 3 x$ for cortical development, the cellular and molecular mechanisms by which $D d x 3 x$ LoF impairs neurogenesis are largely unknown.

DDX3X is a cytoplasmic RNA helicase that promotes translation of mRNAs with highly structured 5' UTRs (Oh et al. 2016; Calviello et al. 2021). Thus, an intriguing possibility is that DDX3X controls cortical development by regulating translation. Although DDX3X translational targets have been identified in immortalized cells (Oh et al. 2016; Calviello et al. 2021), they have not been investigated in neural cells in vivo. Further, while translational control is essential for corticogenesis (Kraushar et al. 2014; Yang et al. 2014; Blair et al. 2017; Zahr et al. 2018; Hoye 
and Silver 2021), there is limited genome-wide assessment of translation in the developing cortex, which restricts our understanding of this important layer of post-transcriptional regulation.

In order to understand how $D d x 3 x$ LoF impairs cortical development at a cellular and molecular level (Fig. 1B), fundamental questions need to be addressed. In which cells and when is DDX3X required during cortical development? What are the sex-specific dosage requirements of $D d x 3 x$ ? What are the key translational targets of DDX3X in the developing brain? In this study we use mouse genetics, live imaging of neural progenitors, and ribosome profiling, to discover underlying cellular and molecular mechanisms for how $D d x 3 x$ LoF impairs cortical development.

\section{Results}

\section{Conditional knockout of $D d x 3 x$ in neural progenitors causes microcephaly and profound} apoptosis

To understand how $D d x 3 x$ LoF impairs cortical development in vivo, we employed a previously generated floxed $D d x 3 x$ mouse (Chen et al. 2016a) and crossed it to Emx1-Cre (Gorski et al. 2002; Chou et al. 2009). This strategy removes $D d x 3 x$ from neural progenitors beginning at E9.5, as well as their progeny. As $D d x 3 x$ is X-linked, we generated Emx1-Cre conditional knockout $(\mathrm{cKO})$ females $\left(D d x 3 x^{10 x / 10 x}\right)$ and males $\left(D d x 3 x^{10 x / Y}\right)$ and conditional heterozygous (cHet) females $\left(D d x 3 x^{10 x /+}\right)$. To verify $D d x 3 x$ mRNA levels were reduced, we performed single molecule inexpensive fluorescence in situ hybridization (smiFISH) (Tsanov et al. 2016) at E12.5 (Fig. 1C). There were visibly less $D d x 3 x$ mRNA puncta in cHet females than in controls and almost no $D d x 3 x$ mRNA puncta in cKO males and females, demonstrating that $D d x 3 x$ levels are reduced.

We then quantitatively assessed $D d x 3 x$ levels in male and female embryonic brains. Towards this, we used the Cre reporter, Ai14 $4^{\text {lox/lox }}$, along with FACS to isolate TdTomato+ cells from embryos, followed by RT-qPCR for $D d x 3 x$. At E11.5, Ddx3x mRNA levels were $\sim 30 \%$ reduced in cHet females, $\sim 40 \%$ reduced in cKO males and $\sim 70 \%$ reduced in cKO females relative to sex-matched controls (Fig. 1D, E). At E14.5, we quantified a similar degree of reduction of 
$D d x 3 x$ in mutant brains (Fig. 1F, G). The incomplete reduction of $D d x 3 x$ in females could reflect inefficiencies in recombination or preferential loss of mutant cells. Notably, control females expressed $\sim 30 \%$ higher levels of $D d x 3 x$ than control males (Fig. 1H). This significant difference is consistent with previous findings in mice and humans (Wu et al. 2014; Tukiainen et al. 2017) and suggests $D d x 3 x$ partially escapes $\mathrm{X}$ chromosome inactivation $(\mathrm{XCl})$ in the embryonic cortex. In all, these data demonstrate divergent $D d x 3 x$ levels in males and females in the developing brain. Further, this establishes Emx1-Cre $D d x 3 x$ conditional mice as a model to interrogate dosedependent and sex-specific requirements of $D d x 3 x$ throughout cortical neurogenesis.

We next assessed gross cortical anatomy and size in $D d x 3 x$ mutant embryos. Strikingly, homozygous loss of $D d x 3 x$ from neural progenitors and their progeny led to profound microcephaly in cKO females at postnatal day 0 (P0) (Fig. 1I, J). The decrease in cortical area began at E14.5, and by P0 was reduced by $50 \%$ (Fig. S1A). In contrast, no significant reduction in cortical area was observed in cHet females or cKO males (Fig. 1I, J). These data demonstrate that the developing brain is highly sensitive to $D d x 3 x$ levels.

As microcephaly is often associated with massive cell death, we assessed apoptosis in $D d x 3 x$ cKO females by immunostaining E12.5 and E14.5 sections for the apoptotic marker, cleaved caspase-3 (CC3). We observed $\sim 5 \%$ of cells in cKO females were CC3+ at E12.5 and 15\% were CC3+ at E14.5 (Fig. S1B, C; Fig. 1K-M). Apoptotic cells were both Tuj1+ and Tuj1-, suggesting complete $D d x 3 x$ loss causes death of both progenitors and newborn neurons (Fig. S1D). In contrast, and consistent with the absence of microcephaly in cHet females and cKO males, CC3+ cells were not detected at either E12.5 or E14.5 in these genotypes (Fig. S1B, C; Fig. 1K-M). Altogether, these data suggest widespread apoptosis is likely a substantial contributor to the reduced cortical area in cKO female mice and further reinforces the notion that the developing murine cortex is exquisitely sensitive to $D d x 3 x$ levels.

The extensive apoptosis in cKO females could be due to $D d x 3 x$ requirements in neural precursors, or alternatively independent requirements in newborn neurons. To understand how 
$D d x 3 x$ loss impacts neuronal survival, we generated $N e x-C r e$, cKO females (Nex-Cre, $\left.D d x 3 x^{\text {lox/lox }}\right)$ (Goebbels et al. 2006). Ddx3x depletion was validated using smiFISH, which showed specific depletion in the cortical plate where neurons reside (Fig. S1E). Unlike Emx1-Cre cKO females, we did not observe microcephaly or apoptosis in Nex-Cre, cKO females across cortical development (E13.5, E15.5, E17.5 (not shown) and P0; Fig. S1F-H). This suggests that apoptosis of newborn neurons in the Emx1-Cre model is due to defects in neural precursors. Taken together, these data reinforce the significant role of $D d x 3 x$ in neural progenitors.

\section{$D d x 3 x$ loss is sexually dimorphic and DDX3Y can compensate for loss of DDX3X}

While the vast majority of individuals with $D D X 3 X$ syndrome are females, males can harbor either maternally inherited or de novo DDX3X mutations (Kellaris et al. 2018; Nicola et al. 2019). Thus, as males only have a single copy of $D d x 3 x$, we predicted that loss of $D d x 3 x$ in males would phenocopy cKO females. Surprisingly, cKO males did not display profound microcephaly or apoptosis (Fig. 1I-M). Notably, males have a $D d x 3 x$ paralog, $D d x 3 y$, on the $Y$ chromosome which can compensate for $D d x 3 x$ at the translational level in immortalized cells (Venkataramanan et al. 2021). Thus, we postulated that $D d x 3 y$ may offset the loss of $D d x 3 x$ in the neocortex. We first examined if $D d x 3 y$ levels were altered following $D d x 3 x$ reduction. In E11.5 $D d x 3 x$ cKO male cortices, Ddx3y mRNA levels were significantly elevated 1.4 fold on average (Fig. 2A). This suggests there is a transcriptional adaptation of $D d x 3 y$ in response to reduced $D d x 3 x$ in the embryonic cortex, consistent with findings in $D d x 3 x$ cKO hindbrains (Patmore et al. 2020).

We next probed the functional redundancy of DDX3X and DDX3Y by investigating requirements for $D d x 3 y$ during cortical development. To this end, we performed in utero electroporation (IUE) of E14.5 brains with Ddx3y sgRNA + Cas9 and pCAG-GFP to deplete Ddx3y in males (Fig. 2B). Using FACS to isolate GFP+ cells from E17.5 brains, we quantified an average $53 \%$ reduction in $D d x 3 y$ mRNA levels following $D d x 3 y$ CRISPR-based depletion; there was no 
effect upon $D d x 3 x$ mRNA expression (Fig. 2C). This indicates that $D d x 3 y$ sgRNAs are specific and effective.

We next assessed the requirement of $D d x 3 y$ for neurogenesis. In control E17.5 male brains (no sgRNA) GFP-positive cells were distributed fairly evenly across cortical bins (Fig. 2D, E). Conversely, $D d x 3 y$ knockdown led to the accumulation of GFP+ cells in the VZ, with few GFP+ cells in the CP (Fig. 2D, E). This defect could be due to impaired migration and/or altered production of neurons. Quantification of GFP+Sox2+ progenitors and GFP+Neurod2+ neurons showed that $D d x 3 y$ depletion led to significantly more progenitors and fewer neurons, as compared to control (Fig. 2F-H). Importantly, these findings phenocopy acute $D d x 3 x$ knockdown by IUE in the embryonic brain (Lennox et al. 2020). This demonstrates that $D d x 3 x$ and $D d x 3 y$ have similar requirements for neurogenesis, suggesting that $D d x 3 y$ partially compensates for $D d x 3 x$ in cKO males. This finding may explain the divergent phenotypes of cKO male and female mice, as well as why some $D D X 3 X$ mutations in human males are tolerated.

\section{$D d \times 3 x$ cHet female and cKO male brains have more progenitors and fewer neurons}

Because $D D X 3 X$ syndrome females are heterozygous and males are hemizygous, we focused our analyses on cHet females and cKO males. Importantly, both genotypes have similar $30-40 \%$ reduction in $D d x 3 \times$ mRNA and normal brain size (Fig. 1). Moreover, redundant functions of DDX3X and DDX3Y, along with transcriptional upregulation of $D d x 3 y$ in cKO males likely equalizes total DDX3 levels in cHet females and cKO males. This provides a rationale for evaluating both sexes to investigate requirements of $D d x 3 x$ in cortical development. For simplicity, going forward we collectively refer to the cHet females and cKO males as $D d x 3 x$ depleted.

Our data using both Emx1-and Nex-Cre drivers suggests that $D d x 3 x$ LoF impairs cortical development by specifically controlling progenitors (Fig. 1). We thus quantified progenitors in Emx1-Cre, $D d x 3 x$ depleted brains at E13.5 and E14.5, stages at which both RGCs and IPs are abundant. Compared to E13.5 control mice, $D d x 3 x$ depleted brains showed similar numbers of 
IPs, although RGCs trended higher (Fig. S2A, B, C). However, by E14.5, the number of RGCs and mature IPs was significantly increased in $D d x 3 x$ depleted brains (Fig. 3A-C). Moreover, there was a concomitant trend towards fewer Tbr2-Sox2- cells following Ddx3x depletion at both E13.5 and E14.5, indicating fewer neurons (Fig. S2D, E). These alterations in cell composition did not significantly impact cortical thickness at E14.5, consistent with the lack of microcephaly in these mice (Fig. 1, S2F).

We next assessed how these cell composition differences ultimately impact excitatory neuron number and laminar organization in P0 brains. We quantified Tbr1 (Layer VI), Ctip2 (Layer V), and Lhx2 (Layer II/III), which all showed slight reductions in Ddx3x depleted mice (Fig. 3DG). However, laminar distribution was unaffected, suggesting that $D d x 3 x$ is largely dispensable for neuronal migration (Fig. S2G, H). These data are consistent with a requirement of $D d x 3 x$ for proper neuron number across all stages of corticogenesis.

\section{$D d x 3 x$ conditional heterozygous progenitors exhibit a longer cell cycle and undergo less neurogenic divisions}

We next aimed to understand the cell biological mechanisms by which $D d x 3 x$ depletion impairs progenitor and neuron number. One possibility is that $D d x 3 x$ depleted progenitors re-enter the cell cycle rather than exiting and terminally differentiating. To investigate this, we quantified cell cycle exit in E14.5 Ddx3x depleted embryos, using a 24 hour pulse of the nucleotide analog, EdU, at E13.5. EdU+Ki67- cells have exited the cell cycle and terminally differentiated, whereas $\mathrm{EdU}+\mathrm{Ki} 67+$ cells are still progressing through the cell cycle. At E14.5, there were significantly more EdU+Ki67+ cells in $D d x 3 x$ depleted brains relative to control (Fig. 4A-C), indicating that $D d x 3 x$ is required for progenitor cell cycle exit.

In order to investigate whether $D d x 3 x$ depletion impaired cell cycle exit of RGCs, IPs, or both, we quantified EdU+ RGCs and IPs using Sox2 and Tbr2, respectively. We measured significantly more EdU+Sox2+ cells in $D d x 3 x$ depleted brains compared to control, indicating 
reduced cell cycle exit of RGCs (Fig. 4D, S3A,B). We also observed a trend towards decreased cell cycle exit of immature IPs (EdU+Sox2+Tbr2+ cells), but no change in mature IPs (Fig. S3C,D). Moreover, there was a significant concomitant decrease in EdU+Sox2-Tbr2- cells in $D d x 3 x$ depleted brains relative to control (Fig. 4E), suggesting that $D d x 3 x$ depletion perturbs generation of neurons. Overall, these data demonstrate that $D d x 3 x$ depletion impairs cell cycle exit, predominantly in RGCs, resulting in generation of fewer neurons.

Based on these results, we posited that DDX3X controls neuron generation by impacting how progenitors divide. Indeed, DDX3X has a conserved role in regulating cell cycle in other contexts ( $\mathrm{Li}$ et al. 2014; Chen et al. 2016a; Kotov et al. 2016; Heerma van Voss et al. 2018). To directly test whether $D d x 3 x$ depletion affects cortical progenitor cell cycle, we employed an established semi-cumulative labeling strategy (Fig. 4F) (Quinn et al. 2007). Briefly, at E14.5, EdU was injected intraperitoneally followed by a BrdU injection 1.5 hours later. At $t=2$ hours, embryonic brains were harvested and Ki67, EdU and BrdU were quantified. Strikingly, the $D d x 3 x$ depleted brains exhibited a significantly longer cell cycle (Tc) than control, nearly 1.5-fold longer (average $17 \mathrm{hr}$ in ctrl vs $25 \mathrm{hr}$ in cHet F/cKO M) (Fig. 4G, H). However, Ddx3x depletion did not specifically impair S phase duration as Ts/Tc was not significantly different (Fig. 4I). Further, there was no significant difference in the percentage of phospho-histone3 $(\mathrm{PH} 3)+\mathrm{Sox} 2+$ cells between controls and $D d x 3 x$ depleted brains (Fig. S3E, F) indicating that G2/M length of RGCs is not specifically affected. Altogether, these data demonstrate that $D d x 3 x$ depletion prolongs overall cell cycle in vivo, without a clear bias towards any specific phase.

We next employed clonal live imaging to monitor both progenitor cell cycle duration and progeny generation (Pilaz et al. 2016). Primary cultures were produced from E14.5 cHet females, cKO males, and controls, and progenitors were live imaged (Fig. 5A). After 24 hours, we fixed and stained cells to monitor direct progeny (Fig. 5A, B). We did not observe any significant differences in mitosis duration (Fig. S3G), which aligns with PH3 quantification at E14.5 (Fig. S3E, F). Consistent with a prolonged cell cycle duration, $D d x 3 x$ depleted cells underwent 
significantly fewer re-divisions relative to controls (Fig. 5C-D). These data are consistent with the longer cell cycle measured in vivo, demonstrating that progenitors in vitro reflect in vivo phenotypes.

Because $D d x 3 x$ depleted brains contain fewer neurons, we also used live imaging to determine whether $D d x 3 x$ loss independently impairs the ability of progenitors to directly produce neurons. Following live imaging, cells were immunostained with Sox2, Tbr2, Tuj1 and Hoechst to discriminate between proliferative, asymmetric neurogenic, and symmetric neurogenic divisions

(Fig. 5B). $D d x 3 x$ depleted progenitors underwent significantly more proliferative $(\mathrm{P}, \mathrm{P})$ divisions and significantly fewer symmetric neurogenic $(\mathrm{N}, \mathrm{N})$ divisions relative to controls (Fig. 5E). Asymmetric neurogenic (N,P) divisions were unchanged. Consistent with CC3 staining at E14.5, there were virtually no apoptotic progeny in cHet females/cKO males and controls. These data demonstrate that, in addition to prolonged cell cycle, $D d x 3 x$ deficient progenitors undergo more proliferative divisions and fewer symmetric neurogenic divisions.

Overall, these findings reveal that DDX3X controls neuron generation by acting in progenitors via two mechanisms. First, progenitor cell cycle duration is increased and progenitors are delayed in cell cycle exit, and second, progenitors that do exit the cell cycle tend to produce progenitors rather than neurons. This provides mechanistic explanation for how $D d x 3 x$ controls cortical neuron generation.

\section{Ribosome Profiling uncovers the translatome of E11.5 neural progenitors}

We next sought to understand molecular mechanisms by which $D d x 3 x$ alters progenitor fate decisions and impairs neurogenesis. DDX3X is an RNA helicase with canonical requirements for translation initiation, particularly for mRNAs with structured 5' UTRs (Calviello et al. 2021). Neural progenitors can be transcriptionally primed which is thought to promote generation of specific cell fates (Li et al. 2020; Hoye and Silver 2021). However, a lack of genome-wide translational data in the developing cortex has limited our understanding of how translational 
control influences neurogenesis. To investigate translation at the earliest stages of cortical development and to identify which mRNAs require DDX3X for their translation, we performed ribosome profiling (Ribo-seq) (Ingolia 2016) and RNA-seq using E11.5 microdissected cortices from cKO and control males and females (Fig. 6A). To ensure maximal $D d x 3 x$ depletion and have the highest sensitivity for identifying DDX3X-dependent translation targets, we focused on cKO females and males. Further, we employed Ribo-seq at E11.5 to avoid confounds due to apoptosis in the cKO females. At this stage, the brain is also largely homogenous composed of mainly neural progenitors. We optimized Ribo-seq on E11.5 cortices (see Materials and Methods) and performed extensive quality control to ensure that ribosome-protected fragments (RPFs) were the correct size (Fig. S4A, B), mapped to the coding region as expected (Fig. S4C), and were in the correct reading frame (Fig. S4D; see methods).

We first examined translational regulation of wildtype progenitors. Overall, we observed a correlation between RNA and RPF abundance (Spearman r=0.9525) (Fig. S4E). Using translation efficiency (TE, a metric reflecting translation per mRNA), taking into account both transcript levels (RNA-seq) and ribosome occupancy (Ribo-seq) (see methods), we assessed whether transcripts are translationally regulated in wildtype E11.5 progenitors. Towards this, we focused on TE of canonical mRNAs known to be enriched in RGCs, IPs, and deep (VI-V) and superficial layer (IVII) neurons (Telley et al. 2019; Di Bella et al. 2021) (see Supplemental Excel 3) as compared to all other genes. We expected that RGC-enriched genes would be highly expressed and translated since RGCs are a predominant cell type at E11.5. IPs and deep layer neurons are produced beginning at E11.5 and E12.5, respectively. In contrast, superficial layer neurons are born later (E13.5-E17.5), and thus, we expected lower translation of these genes. To our surprise we found that RGC-, IP-, and deep layer neuron-enriched transcripts had significantly higher TE than the average transcript expressed at this stage (other genes), consistent with translational upregulation (Fig. 6B). Moreover, there was a notable divergence between deep (positive TE) and superficial (negative TE) layer neuron transcripts (Fig. 6B), suggesting superficial layer 
neuron transcripts are translationally repressed. This suggests that in addition to transcriptional priming, progenitors also use translational priming to direct cell fate. Moreover, these results indicate that observed differences in TE might reflect developmental stepwise translational repression.

Ribo-seq can also reveal the use of upstream open reading frames (uORFs), which frequently cause translational repression of the downstream, canonical ORF (Johnstone et al. 2016). We thus used ORFquant to assess canonical ORFs and uORFs in cortical progenitors (Calviello et al. 2020). This revealed $\sim 14,000$ annotated ORFs and $\sim 2500$ uORFs, including a prominent uORF in the lissencephaly gene, Pafah1b1 or Lis1 (Fig. 6C). We also identified 1200 ORFs in non-coding RNAs, including a novel ORF in the Rab26os IncRNA (Fig. 6C, S4F, G). Thus, this rich dataset provides a valuable resource to interrogate the use of uORFs during cortical development and suggests an important mode of gene expression regulation in the developing cortex. Overall, these high-quality Ribo-seq data will enable the generation of novel hypotheses regarding translational control and cortical development.

\section{DDX3X-dependent translation targets are critical for neurogenesis}

We then turned to our $D d x 3 \times$ cKO data to discover DDX3X-dependent translation targets and identified 188 targets that had differential TE (p-adjusted <0.1) (Fig. 6D). The low number of DDX3X translation targets is on-par with translational studies of DDX3X in immortalized cells and reinforces that DDX3X is not a general translation factor (Calviello et al. 2021). Virtually all targets (183 of 188) had a lower TE in cKO mice, consistent with DDX3X promoting translation. About half of these TE changes were driven by changes in the input RNA levels while the other half had significantly lower TE with little to no change in the corresponding input RNA. We thus focused on the 87 targets in which the input RNA was $<0.5 \log 2 \mathrm{FC}$, as these are most likely to be bona fide DDX3X translation targets (Fig. 6D, E). Rcor2, Setd3, and Topbp1, were amongst those targets showing a general decrease in RPFs along the mRNA (Fig. 6F). 
To orthogonally evaluate these DDX3X-dependent translational targets we employed polysome fractionation using E14.5 cortices of cHet females and cKO males, as well as controls (Fig. 6G). We chose this later stage to assess targets which may reflect or explain cell composition changes at E14.5 (Fig. 3A-C). Therefore, we isolated RNA from the monosome and high molecular weight (HMW) polysome fractions and performed RT-qPCR of putative DDX3X translation targets. Both Rcor2 and Setd 3 were significantly enriched in the monosome fraction and significantly depleted from the polysome fraction for cHet females/cKO males as compared to controls (Fig. 6I, J), consistent with their decreased TE at E11.5. Topbp1 was significantly depleted from the polysome fraction, but unchanged in the monosome fraction in cHet females/cKO males relative to controls (Fig. 6K). Another putative target, Hax1, showed trends to shift from polysomes (Fig. S4F). Interestingly, all of these putative targets were also significantly upregulated in the input RNA at E14.5 (Fig. 6H), even though we selected targets whose RNA levels were normal in E11.5 mutant brains. This could suggest a feedback mechanism, whereby over time DDX3X-dependent targets are transcriptionally upregulated in response to their impaired translation. These shifts of DDX3X-dependent translation targets towards monosomes and concomitant depletion from polysomes were specific. For example, $\beta$ actin levels, were comparable between monosome fractions ( $\beta$-actin CT average 25.06 vs 24.58) and polysome fractions ( $\beta$-actin CT average 22.73 vs 22.72 ) of cHet females/cKO males and controls. Altogether the shift towards monosomes along with reduced TE reflects an essential requirement of DDX3X for translation of specific mRNAs in cortical progenitors in vivo.

We next asked whether DDX3X translational targets have known roles in neurogenesis. RCOR2 is critical for neural proliferation (Wang et al. 2016; Monaghan et al. 2017), and Topbp1 deletion in neural progenitors results in DNA damage and apoptosis (Lee et al. 2012). These requirements are notably similar to $D d \times 3 \times$ LoF, indicating that DDX3X-dependent translational targets are critical within neural progenitors for cortical development. 
Another DDX3X dependent target, Setd3, is expressed in the developing cortex (Telley et al. 2019), but its requirements for corticogenesis are unknown. SETD3 is a histidine methyltransferase, which may modify histone H3, as well as actin H73 (Witecka et al. 2021). Consistent with this, Setd3 is implicated in cancer progression and cell cycle control, as well as cytoskeletal integrity (Witecka et al. 2021). To test the requirements of Setd3 for cortical development, we validated siRNAs to knockdown Setd3 in N2A cells and observed a $\sim 50 \%$ reduction after 24 hours (Fig. 7A). We then performed IUEs to deliver either scrambled or Setd3 siRNAs, along with pCAG-GFP, at E14.5 and collected embryos at E17.5. Setd3 knockdown significantly altered the distribution of GFP+ cells, with significantly more cells in the VZ and fewer cells in the CP (Fig. 7B, C). Moreover, Setd3 knockdown resulted in more Sox2+ RGCs and fewer Neurod2+ neurons (Fig. 7D), phenocopying $D d x 3 x$ LoF. This suggests that decreased TE of Setd3, as well as other DDX3X-dependent targets, may synergistically contribute to Ddx3x LoF neurogenesis phenotypes (Fig. 7E). Taken together these data show that DDX3X controls translation of a repertoire of genes which are collectively critical in neural progenitors for neurogenesis.

\section{Discussion}

Mutations in $D D X 3 X$ are a leading cause of ID, as well as other developmental phenotypes, which are classified as DDX3X syndrome (Johnson-Kerner et al. 1993; Snijders Blok et al. 2015; Wang et al. 2018; Beal et al. 2019; Lennox et al. 2020). Half of the over 100 known $D D X 3 X$ mutations are nonsense, predicted to act in a LoF manner. Yet, the molecular and cellular mechanisms by which $D d x 3 x$ LoF perturbs cortical development have been largely unknown. Here, we leveraged a new genetic model of $D d x 3 x$ LoF to discover that DDX3X promotes neuronal generation by regulating the length and fate of progenitor divisions, namely RGCs and IPs. We further use in vivo Ribo-seq to define the repertoire of transcripts whose translation depends upon DDX3X, many of which are critical for neurogenesis. Our results provide invaluable 
new insights into the underpinnings of $D D X 3 X$ syndrome and highlight candidate targets and possible pathways for therapeutic intervention.

\section{$D d x 3 x$ dosage underlies cell fate and sexually dimorphic phenotypes}

While the vast majority of $D D X 3 X$ syndrome individuals are female, an increasing number of male individuals have been identified (Kellaris et al. 2018; Nicola et al. 2019), comprising 5\% of all cases (ddx3x.org). Using our conditional mouse model, we interrogated phenotypic overlap in males and females, as well as the role of the paralog $D d x 3 y$. Complete loss of $D d x 3 x$ led to microcephaly in females, but not in hemizygous males, suggesting that brain development is sexually dimorphic. We posit that $D d x 3 y$ expression explains why cKO male mice are phenotypically milder than cKO females; we found that $D d x 3 y$ loss phenocopies $D d x 3 x$ neurogenesis defects. Likewise, in both the hindbrain and hematopoietic system, $D d x 3 y$ can also compensate for loss of $D d x 3 x$ in vivo (Szappanos et al. 2018; Patmore et al. 2020). The ability of $D d x 3 y$ to compensate for $D d x 3 x$ loss may be due in part to redundant translational regulation (Venkataramanan et al. 2021), as well as transcriptional adaptation of $D d x 3 y$ in response to $D d x 3 x$ loss (Fig. 2). Taken together these data provide a possible explanation for why males carrying $D D X 3 X$ mutations are viable (Kellaris et al. 2018; Nicola et al. 2019; Lennox et al. 2020). Because $D d x 3 x$ partially escapes $\mathrm{XCI}$ in females (Fig. 1), we hypothesize that DDX3Y normalizes total DDX3 levels between males and females; this may explain why cHet females and cKO males have comparable phenotypes.

In addition to being sexually dimorphic, we discover that neural cells of the developing cortex are exquisitely sensitive to $D d x 3 x$ levels, as loss of one versus two copies causes vastly different corticogenesis phenotypes. In the case of cKO females, there is profound apoptosis in progenitors and neurons, whereas cHet females and cKO males have impaired neurogenesis without cell death. This sensitivity of the developing brain to $D d x 3 x$ dosage is highly relevant for 
interpreting the spectrum of nonsense mutations, as some of these may be LoF whereas others may be hypomorphic.

\section{$D d x 3 x$ is required for neural progenitor cell division which underlies cortical abnormalities}

We show that DDX3X controls the function of neural progenitors and generation of neurons across all laminar layers throughout cortical development through two key mechanisms. First, $D d x 3 x$ depletion increases progenitor cell cycle duration by 1.4 fold. It does so without altering any specific phase, consistent with a study of DDX3X in cancer cells using $\mathrm{FUCCI}$ (Heerma van Voss et al. 2018). Additionally, $D d x 3 x$ depleted progenitors in vitro underwent fewer re-divisions in a 24-hour period; if this increase in cell cycle duration were sustained over the course of neurogenesis, we predict it would result in overall fewer progenitor divisions, and ultimately fewer neurons (Takahashi et al. 1995). Consistent with this, neurons were reduced across all layers at P0. Second, $D d x 3 x$ controls the balance of proliferative versus neurogenic divisions. This is particularly fascinating as it demonstrates that DDX $3 X$ functions in progenitors to direct neural fates of daughter progeny. Our Ribo-seq suggests that these cellular mechanisms might be influenced by translational control in progenitors.

Going forward, it will be important to further dissect how DDX3X dosage influences neural progenitor cell cycle and cell fate. $D d x 3 x$ depletion had a particularly potent impact on RGCs, relative to IPs, suggesting that RGCs may be especially sensitive to loss of DDX3X, which is consistent with higher $D d x 3 x$ expression in RGCs relative to IPs (Telley et al. 2019). Moreover, this could reflect differences in cell cycle duration of these progenitors (Arai et al. 2011).

Using a conditional mouse model provided distinct advantages to propel our understanding of $D d x 3 x$ requirements during cortical development. New insights into $D d x 3 x$ dosage requirements and redundant roles of DDX3X and DDX3Y would not have been gained with germline LoF models, due to male lethality (Chen et al. 2016a). A recent report assessed behavioral phenotypes of a germline mouse model of $D d x 3 x$ haploinsufficiency in females 
(Boitnott et al. 2021). Consistent with our cHet phenotypes, these females were not microcephalic, but did have reduced Ctip2+ (layer $\mathrm{V}$ ) neurons in the somatosensory cortex at P3, which persisted into adulthood. These germline mice have overall gross delay, defects in motor coordination, and sensory behavioral abnormalities. Our study provides valuable insights to explain the embryonic underpinnings of these behavioral deficits.

We also gained new insights into cell-autonomous $D d x 3 x$ requirements in brain development. Using a Nex-Cre cKO mouse model, we found that $D d x 3 x$ is largely dispensable in newborn neurons for their migration and survival, indicating that observed neuronal loss largely arises from impairments in neural progenitors. However, $D d x 3 x$ does control neurite outgrowth (Chen et al. 2016b) and is a component of neuronal transport granules (Kanai et al. 2004; Elvira et al. 2006), suggesting it could function in mature neurons. DDX3X likely functions in other CNS cell types, as well, as $50 \%$ of $D D X 3 X$ syndrome individuals present with white matter loss (Lennox et al. 2020), which could arise from impaired gliogenesis. Indeed, Perturb-seq of 30 ASD-risk genes found that $D d x 3 x$ depletion alters oligodendrocyte gene expression (Jin et al. 2020). Moreover, $D D X 3 X$ also regulates innate immunity (Szappanos et al. 2018), raising the intriguing idea that $D d x 3 x$ could function in microglia to influence cortical development. Thus, roles for $D d x 3 x$ in glial cells and mature neurons should be explored further.

\section{Translation during cortical development and DDX3X-dependent translational control of progenitors}

In the neurodevelopment field there is a wealth of transcriptomic data, but far less translation/proteomic data for embryonic mouse cortex. Using Ribo-seq, we have generated a valuable new resource for understanding translation at the onset of neurogenesis. To our knowledge, this is one of the first reports to assess wildtype translation using Ribo-seq at the onset of neurogenesis, with exception of a recent pre-print which examined later stages of development (Harnett et al. 2021). scRNAseq studies have revealed transcripts important for 
deep and superficial layer neuronal fates are expressed in RGCs (Telley et al. 2019); however it's generally thought that these are translationally repressed until neurons are born. Intriguingly, our data show that deep layer transcripts have a higher TE than superficial layer transcripts, though less than RGCs and IPs. This suggests that there could be translational priming of progenitors, in addition to transcriptional priming. Given the instrumental role that translational factors play in cortical development (Kraushar et al. 2014; Yang et al. 2014; Zahr et al. 2018; Hoye and Silver 2021), our data thus provides valuable targets which can be functionally tested at neuroepithelial stages and beyond.

Although DDX3X is a known translation regulator (Oh et al. 2016; Calviello et al. 2021), a key gap has been the identification of its targets in the developing brain. Using Ribo-seq, we discovered DDX3X-dependent targets in neural progenitors in vivo. Both Rcor2 and Topbp1 have known roles in cortical neurogenesis and we further established new requirements for Setd3. Both Rcor2 and Setd3 are epigenetic regulators (Wang et al. 2016), suggesting their decreased TE may alter gene expression and cell fate. Notably, epigenetic regulators can influence gene expression by complexing with transcription factors. Indeed, RCOR2 binds to Insulinomaassociated 1 (Monaghan et al. 2017), a transcription factor required in IPs for neurogenesis (Farkas et al. 2008). DDX3X-mediated epigenetic regulation will be fascinating to investigate in future studies, especially considering that many epigenetic regulators and chromatin-modifying enzymes are implicated in ID and ASD (Grafodatskaya et al. 2010). In sum, our translational analysis highlights new downstream pathways relevant for DDX3X function and disease pathology.

We speculate that reduced TE of DDX3X-dependent transcripts collectively contributes to phenotypes in mouse models and perhaps in human $D D X 3 X$ syndrome. Given that $D d x 3 x$ LoF modestly affects TE of many transcripts in neural progenitors, we do not anticipate that increasing TE of any one transcript would alleviate $D d x 3 x$ LoF phenotypes. However, understanding how DDX3X is recruited to these transcripts to specifically promote translation initiation might uncover 
valuable mechanisms by which transcripts with low TE could be boosted translationally for therapeutic intervention. Likewise, elucidating RNA structures of these targets might lead to development of therapeutic small molecules which can overcome DDX3X-dependent translation.

Interestingly, DDX3X-dependent translation targets were shifted from polysomes to monosomes, consistent with reduced TE and a role for DDX3X in translational initiation. However, the basal RNA expression of DDX3X targets was also higher at E14.5 compared to E11.5. This suggests that reduced TE of DDX3X-dependent targets leads to a feedback mechanism whereby these mRNAs are transcriptionally upregulated over time. This potential feedback loop is relevant for those pursuing Ribo-seq experiments to consider, as putative targets are typically defined by RPF density changes but not the input RNA (Ingolia 2016).

\section{The landscape of DDX3X mutations with divergent phenotypes}

Our work further substantiates the importance of post-transcriptional RNA regulation in cortical development and disease. There is a strong association between mutations in translation regulators and neurodevelopmental disorders (Chen et al. 2019; Hoye and Silver 2021). As we have shown, investigation of DDX3X and translational regulators can give valuable insights into the etiology of these disorders by discovering networks of neurogenesis regulation including crucial factors, such as SETD3.

Our work also provides a mechanistic understanding of how $D d x 3 x$ LoF impairs embryonic cortical development; this may model human $D D X 3 X$ nonsense mutations that result in haploinsufficiency. The extent to which these mechanisms extend to all mutations is unknown. Indeed, it is imperative to define how the full spectrum of $D D X 3 X$ mutations impact its expression and ultimately, influence corticogenesis. Half of $D D X 3 X$ mutations are missense, with a subset showing more clinically severe outcomes relative to those carrying nonsense mutations (Lennox et al. 2020). Interestingly, $D D X 3 X$ missense mutations might differentially impair translation of DDX3X targets (Lennox et al. 2020; Calviello et al. 2021). Thus, diverse DDX3X mutations may 
result in divergent molecular and cellular pathologies and our study provides an important foundation upon which future investigations of $D D X 3 X$ mutations may be compared.

\section{Materials and Methods}

Mouse husbandry

All animal use was approved by the Duke Division of Laboratory Animal Resources and the Institutional Animal Care and Use Committee. The following lines were used and genotyped as described, all on C57BL/6J background: Emx1-Cre (005628) (Gorski et al. 2002) and Ai14 (007914) (Madisen et al. 2010) (Jackson Laboratory); Nex-Cre (Goebbels et al. 2006) (gift, KlausNave); $D d x 3 x^{10 x / / 0 x}$ (Chen et al. 2016a) (gift, Li-Ru You). Plug dates were defined as E0.5 on the morning the plug was identified.

\section{Statistical methods and rigor}

Exact statistical tests, $\mathrm{p}$-values, and $\mathrm{n}$ for each analysis are reported in Supplemental Excel 1. For each experiment, both male and female mice were used and littermates were used when possible. All analyses were performed by 1 or more blinded investigators.

\section{Primary cultures and live imaging}

Primary cortical cultures were derived from E14.5 embryonic dorsal cortices, as described (Mitchell-Dick et al. 2019), but with minor modifications: 1) cortices were trypsinized for 6 minutes and 2) 150,000 cells were plated on poly-D-lysine coated glass-bottom 24 well culture plates (MatTek). Images were captured every 10 minutes and mitosis duration and cell division were identified as previously (Pilaz et al. 2016). Fate determination was performed post-imaging by immunostaining for Tuj1, Sox2, and Tbr2, as described (Mitchell-Dick et al. 2019).

Plasmids, subcloning, and qRT-PCR analysis 
Ddx3y sgRNAs were designed using Benchling and cloned into the pX330-U6-Chimeric_BB-CBhhSpCas9 plasmid (AddGene \# 42230) as described (http://www.addgene.org/crispr/zhang/). cDNA synthesis and qPCR were performed using the iScript Reverse Transcriptase and the iTaq Universal SYBR Green supermix (BioRad), respectively, per manufacturer's instructions. The primers for qRT-PCR all had an annealing temperature of $60^{\circ} \mathrm{C}$ and relative expression was normalized to $\beta$-actin. See Supplemental Excel 2 for sgRNAs and primers.

\section{Immunofluorescence}

Embryonic brains were fixed and sectioned as previously (Mao et al. 2015).. Coronal $20 \mu \mathrm{m}$ sections from the somatosensory cortex were permeabilized with 1 X PBS/0.25\% TritonX-100 and blocked with 5\% NGS/PBS for 1 hour at room temperature. Sections were incubated with primary antibodies overnight at $4^{\circ} \mathrm{C}$, and secondary antibodies at room temperature for 1 hour (Alexa Fluor-conjugated, Thermo Fisher, 1:500). EdU staining was performed as previously (MitchellDick et al. 2019). The following primary antibodies were used, rat: anti-SOX2 (Thermo Fisher, 149811-82, 1:1000), anti-BrdU (Abcam, ab6326, 1:200); mouse: anti-TUJ1 (Biolegend, 801202, 1:2000), anti-CTIP2 (Abcam, c8035, 1:500); rabbit: anti-TBR2 (Abcam, AB23345, 1:1000), antiCC3 (Cell Signaling, 9661, 1:250), anti-NEUROD2 (Abcam, AB104430, 1:500), anti-Ki67 (Cell Signaling Technology, 12202, 1:1000), anti-PH3 (Millipore, 06-570, 1:500), anti-TBR1 (Cell Signaling Technology, 49661S, 1:1000), anti-Lhx2 (Millipore, ABE1402, 1:500), anti-DDX3X (Sigma Aldrich, HPA001648, 1:500); chicken anti-GFP (Abcam, Ab13970, 1:1000). Slides were mounted with Vectashield (Vector Labs, H-1000-10).

\section{Imaging and Analysis}

Images were captured using a Zeiss Axio Observer Z.1 equipped with an Apotome for optical sectioning at 10X, 20X and/or 63X. For each experiment, 2-3 sections were imaged/embryo; images were captured with identical exposures, cropped (200 or $300 \mu \mathrm{m}$ radial columns), and 
brightness was equivalently adjusted across all images in Fiji. Cells were either manually (Fiji cell counter) or automatically (QuPath) counted. For QuPath, the following parameters were adjusted: requested pixel size $=0.1 \mu \mathrm{m}$, background radius $=5 \mu \mathrm{m}$, minimum area $=10 \mu \mathrm{m}^{2}$, maximum area $=$ $200 \mu \mathrm{m}^{2}$, cell expansion $=2 \mu \mathrm{m}$, include cell nucleus and smooth boundaries were unchecked. The threshold was set for each individual channel, but equivalently across all sections (generally between 25-100). For binning analysis, 200 or $300 \mu \mathrm{m}$ wide radial columns were divided into 5 or 10 evenly spaced bins spanning from the ventricular (bin 1) to the pial (bin 5 or 10) surface. Each cell was assigned to a bin to calculate the distribution.

\section{In utero electroporation}

Plasmids were delivered to embryonic brains and IUEs were performed as previously (Lennox et al. 2020). Plasmids were used at the following concentrations: pCAG-GFP $(1.0 \mu \mathrm{g} / \mu \mathrm{L}), \mathrm{pX} 330$ empty or pX330-Ddx3y Ex2 sgRNA (2.4 $\mu \mathrm{g} / \mu \mathrm{L})$. Scrambled (Qiagen, 1022076) or Setd3 siRNAs (Qiagen 1027416, Gene ID: 52690) were injected at $2.5 \mu \mathrm{M}$.

\section{EdU and BrdU injections}

For cell cycle exit, EdU was administered by IP injection at $10 \mathrm{mg} / \mathrm{kg}$ to pregnant dams at E13.5 and embryos were harvested exactly 24 hours later. For semi-cumulative labeling, EdU was administered by IP injection at $10 \mathrm{mg} / \mathrm{kg}$ at $\mathrm{t}=0$ followed by BrdU (30 $\mathrm{mg} / \mathrm{kg})$ at $\mathrm{t}=1.5$ hours. The following calculations were used to derive Tc and Ts: $S$ cells $=$ BrdU+; $P$ cells $=K i 67+; L$ cell fraction=EdU+BrdU- (ie: EdU+ minus BrdU+); Ts=(S cells/L cells $){ }^{*} 1.5 ; \mathrm{Tc}=(\mathrm{P}$ cells $/ \mathrm{S}$ cells $){ }^{*} \mathrm{Ts}$

\section{Single molecule fluorescence in situ hybridization (smFISH)}

smFISH probes against Mus musculus Ddx3x were designed and prepared as described (Tsanov et al. 2016). All solutions and buffers were prepared with diethyl pyrocarbonate, including PFA and sucrose, to quench RNAse activity. $20 \mu \mathrm{m}$ coronal sections were permeabilized in $0.5 \%$ Triton 
$\mathrm{X}-100$ in PBS for $30 \mathrm{~min}$ at room temperature and rinsed twice with buffer containing $10 \%$ formamide and 2x SCC buffer (Thermo 15557044). smFISH probes were diluted 1:200 in buffer containing $10 \%$ formamide, $2 x$ SCC buffer and $10 \%$ dextran sulfate; $200 \mu \mathrm{L}$ of diluted probes were added to each slide and incubated overnight at $37^{\circ} \mathrm{C}$. The next day, samples were washed two times at $37^{\circ} \mathrm{C}$ and Hoechst was included in second wash. Slides were mounted with Vectashield (Vector Labs, $\mathrm{H}-1000-10)$. Slides were imaged at 63X.

\section{FACS}

Samples were processed for FACS as described (Mitchell-Dick et al. 2019) and sorted at $6{ }^{\circ} \mathrm{C}$ using a B-C Astrios cell sorter with gates for forward scatter (FSC), side scatter (SSC), DAPI or PI, GFP or TdTomato, and were sorted directly into RLT buffer and RNA was extracted (Qiagen RNAeasy kit; Qiagen 74034).

\section{RNAseq and Ribosome Footprinting}

Embryonic cortices were flash-frozen in liquid nitrogen and stored at $-80^{\circ} \mathrm{C}$. For each $\mathrm{n}$, four $\mathrm{E} 11.5$ microdissected cortices were thawed on ice and lysed in $400 \mu \mathrm{L}$ of polysome buffer $(20 \mathrm{mM}$ Tris$\mathrm{HCl} \mathrm{pH} \mathrm{7.5,} 150$ mM NaCl, 5 mM MgCl2, 1 mM DTT, 100 mg/ml cycloheximide (CHX; Calbiochem CAS 66-81-9), 25 U/ml Turbo DNase I (Invitrogen AM2238) using a hand-blender. Lysates were pelleted at $2000 \mathrm{~g}$ for 10 mins and titurated with a 26 -gauge needle before adding TritonX-100 to $1 \%$. Lysates were then clarified at $20,000 \mathrm{~g}$ for 10 mins. $100 \mu \mathrm{L}$ of lysate was taken for RNA, to which $300 \mu \mathrm{L}$ of RLT buffer was added and RNA was extracted using the RNAeasy kit (Qiagen, 74034). The remaining lysate was treated with $1 \mu \mathrm{L}$ of RNase I (Ambion AM2294) and incubated for 30 mins at RT. After nuclease digestion, $1 \mu \mathrm{L}$ of Superase In (Invitrogen AM2694) was added, followed by $1 \mathrm{ml}$ of TRIzol (Invitrogen 15596026) and $200 \mu \mathrm{L}$ of chloroform. The samples were vigorously shaken for $30 \mathrm{~s}$ and incubated for 3 minutes at RT before centrifugation at $12,000 \mathrm{~g}$ for 10 mins at $4^{\circ} \mathrm{C}$. $\sim 750 \mu \mathrm{L}$ of supernatant was mixed with $750 \mu \mathrm{L}$ of isopropanol, $2 \mu \mathrm{L}$ of 
glycoblue (Invitrogen AM9515), and $30 \mu \mathrm{L}$ of sodium acetate (NaOAc) and frozen at $-20^{\circ} \mathrm{C}$ overnight. The next day, samples were spun $\left(20,000 \mathrm{~g}, 15 \mathrm{mins}, 4^{\circ} \mathrm{C}\right)$ and the pellet was washed $2 \mathrm{X}$ with $80 \%$ ethanol and allowed to air-dry before resuspending the pellet in $7 \mu \mathrm{L}$ of RNase-free water. A PNK reaction was then performed at $37^{\circ} \mathrm{C}$ for 30 mins on digested RNA by adding $1 \mu \mathrm{L}$ of PNK buffer, $1 \mu \mathrm{L}$ of $100 \mathrm{mM} \mathrm{ATP}$, and $1 \mu \mathrm{L}$ of PNK enzyme (NEB M0247S). During the incubation, a 15\% acrylamide denaturing urea-gel (Biorad 4566053) was pre-run (200V, 15 mins, 1X TBE buffer). Samples (containing $2 X$ sample dye (Novex LC6876) were run ( 45 mins, 200V), along with dsDNA (Thermo 10488023) and miRNA ladders (NEB N2102S). Afterwards, the gel was incubated with SYBR gold (Invitrogen S11494) for 5 mins and the RPFs ( 25-30 nt) were isolated and frozen in $400 \mathrm{mM} \mathrm{NaOAc}$ at $-80^{\circ} \mathrm{C}$. The following day, frozen gel fragments were thawed $\left(95^{\circ} \mathrm{C}, 5 \mathrm{mins}\right)$ and vortexed (20 mins, 3X). The supernatant was spun through a SpinX column (Corning CLS8162) (max speed,10 mins) and $500 \mu \mathrm{L}$ isopropanol and $2 \mu \mathrm{L}$ glycoblue were added before freezing $\left(-20^{\circ} \mathrm{C}, 1 \mathrm{hr}\right)$. The samples were centrifuged (max speed, 15 mins, $4^{\circ} \mathrm{C}$ ) and washed $2 \mathrm{X}$ with $80 \% \mathrm{EtOH}$. The pellet was resuspended in $5 \mathrm{uL}$ of water and libraries were prepared using the Qiagen miRNA library prep kit (331502; followed protocol for $100 \mathrm{ng}$ input and did 15 cycles of PCR amplification). Ribosomal RNA depletion was performed after the 3' adapter ligation step using the RiboMinus Eukaryote kit v2 (Thermo Fisher A15020). For RNAseq cDNA library preparation, RNA concentrations were measured using a NanoDrop spectrophotometer (Thermo Scientific) and the quality of RNA was assessed using an Agilent fragment analyzer and the RNA Analysis Kit (15 nt; Agilent, DNF-471). cDNA libraries were generated using the Kapa mRNA HyperPrep kit with mRNA capture (KapaBiosystems, KR1352) using 50 ng input RNA.

\section{Sequencing and Bioinformatic analysis}

3 samples per condition per sex (WT and cKO) were sequenced, but one WT F had to be removed due to inefficient library prep. RNAseq and Ribo-seq libraries were sequenced on the NovaSeq 
6000 S-Prime with 150 bp paired-end reads and 75 bp single end reads, respectively. For RNAseq, libraries were sequenced to a depth of $\sim 40-60$ million total reads per sample. For Riboseq, libraries were sequenced to a depth of 50-70 million reads and only reads that uniquely mapped were retained. After adapter removal, reads were mapped to rRNA (Langmead, 2012) using bowtie2 v2.4.4, the remaining reads were mapped to GENCODE22 using STAR v2.7.9a (Dobin, 2013). Quality control of mapped reads and count matrices, using uniquely mapping reads only, were obtained using RibosomeProfilingQC (Jianghong Ou 2021) and Ribo-seQC (Calviello et al. 2019). Changes in TE were calculated using DESeq2 by using assay type (RNA-seq or Ribo-seq) as an additional covariate. Translationally regulated genes were defined using an FDR $<0.05$ from a likelihood ratio test, using a reduced model without the assay type covariate, e.g. assuming no difference between RNA-seq and Ribo-seq counts (Calviello, 2021). ORFquant (Calviello et al. 2020) v1.02 was used to de novo identify translated ORFs using the pooled Riboseq data and Gencode M22 annotation as reference. Inputs to ORFquant were obtained using RiboseQC (Calviello et al. 2019). Only ATG-starting ORFs were detected using uniquely mapping reads only.

\section{Polysome Profiling}

Embryonic cortices were dissected and lysed identically to those used for Ribo-seq. Following clarification at $20,000 \mathrm{~g}, 50 \mu \mathrm{L}$ of lysate was taken for RNA input. Clarified lysates were added to prepared $15 \%-50 \%$ sucrose gradients (in the lysis buffer above, except lacking CHX and DNase) and ultracentrifuged $\left(35,000 \times \mathrm{g}, 2\right.$ or 3.5 hours, $\left.4^{\circ} \mathrm{C}\right)$ using a SW41 Ti rotor. Following ultracentrifugation, 12 fractions ( $\sim \mathrm{ml}$ each) were collected from each sample using a BioComp Piston Gradient Fractionator instrument fitted with a TRIAX flow cell to measure absorbance. RNA was extracted from $300 \mu \mathrm{L}$ of each fraction or pooled fractions using TRIzol LS reagent (Thermo Fisher 10296010). cDNA was synthesized from 250 ng of RNA using the iScript cDNA synthesis kit (Bio-Rad) (40 min, $46^{\circ} \mathrm{C}$ ), followed by RT-qPCR using gene-specific primers. Gene expression 
across different fractions was normalized to $B$-actin and then set relative to the corresponding expression from input.

\section{Competing Interests Statement}

The Authors declare no competing interests.

\section{Acknowledgements}

We thank the DDX3X Foundation and members of the Floor, Sherr, and Silver labs for helpful discussions. This work was supported by the Holland-Trice Foundation, the DDX3X Foundation (DLS), NIH R21ND104514 (DLS), NIH R01NS120667 (DLS, SNF); Regeneration Next Initiative Postdoctoral Fellowship, NIH F32NS112566 (MLH); National Institutes of Health DP2GM132932 (SNF). S.N.F. is a Pew Scholar in the Biomedical Sciences, supported by The Pew Charitable Trusts. We thank Chris Nicchitta for help with Ribo-seq and Stacy Horner for use of the polysome profiling equipment. We thank Klaus-Nave (Nex-Cre) and Li-Ru You (Ddx3 $\left.3 x^{10 x / 10 x}\right)$ for mice. We thank the Duke Flow Cytometry and Genomics cores.

\section{Author contributions}

MLH and DLS conceived of and designed the study. MLH and DLS wrote the manuscript. Data was generated/analyzed by MLH, LC, NNE, AJP, CRN, and JO. All authors edited the manuscript. 


\section{References}

Arai Y, Pulvers JN, Haffner C, Schilling B, Nusslein I, Calegari F, Huttner WB. 2011. Neural stem and progenitor cells shorten S-phase on commitment to neuron production. Nat Commun 2: 154.

Beal B, Hayes I, McGaughran J, Amor DJ, Miteff C, Jackson V, van Reyk O, Subramanian G, Hildebrand MS, Morgan AT et al. 2019. Expansion of phenotype of DDX3X syndrome: six new cases. Clin Dysmorphol 28: 169-174.

Blair JD, Hockemeyer D, Doudna JA, Bateup HS, Floor SN. 2017. Widespread Translational Remodeling during Human Neuronal Differentiation. Cell Rep 21: 2005-2016.

Boitnott A, Garcia-Forn M, Ung DC, Niblo K, Mendonca D, Park Y, Flores M, Maxwell S, Ellegood J, Qiu LR et al. 2021. Developmental and Behavioral Phenotypes in a Mouse Model of DDX3X Syndrome. Biol Psychiatry 90: 742-755.

Calviello L, Hirsekorn A, Ohler U. 2020. Quantification of translation uncovers the functions of the alternative transcriptome. Nat Struct Mol Biol 27: 717-725.

Calviello L, Sydow D, Harnett D, Ohler U. 2019. Ribo-seQC: comprehensive analysis of cytoplasmic and organellar ribosome profiling data. bioRxiv: 601468.

Calviello L, Venkataramanan S, Rogowski KJ, Wyler E, Wilkins K, Tejura M, Thai B, Krol J, Filipowicz W, Landthaler $M$ et al. 2021. DDX3 depletion represses translation of mRNAs with complex 5' UTRs. Nucleic Acids Res 49: 5336-5350.

Chen CY, Chan CH, Chen CM, Tsai YS, Tsai TY, Wu Lee YH, You LR. 2016a. Targeted inactivation of murine $\mathrm{Ddx} 3 \mathrm{x}$ : essential roles of $\mathrm{Ddx} 3 \mathrm{x}$ in placentation and embryogenesis. Hum Mol Genet 25: 2905-2922.

Chen HH, Yu HI, Tarn WY. 2016b. DDX3 Modulates Neurite Development via Translationally Activating an RNA Regulon Involved in Rac1 Activation. J Neurosci 36: 9792-9804.

Chen YC, Chang YW, Huang YS. 2019. Dysregulated Translation in Neurodevelopmental Disorders: An Overview of Autism-Risk Genes Involved in Translation. Dev Neurobiol 79: 60-74.

Chou SJ, Perez-Garcia CG, Kroll TT, O'Leary DD. 2009. Lhx2 specifies regional fate in Emx1 lineage of telencephalic progenitors generating cerebral cortex. Nat Neurosci 12: 13811389.

Di Bella DJ, Habibi E, Stickels RR, Scalia G, Brown J, Yadollahpour P, Yang SM, Abbate C, Biancalani T, Macosko EZ et al. 2021. Molecular logic of cellular diversification in the mouse cerebral cortex. Nature 595: 554-559.

Elvira G, Wasiak S, Blandford V, Tong XK, Serrano A, Fan X, del Rayo Sanchez-Carbente M, Servant $F$, Bell AW, Boismenu D et al. 2006. Characterization of an RNA granule from developing brain. Mol Cell Proteomics 5: 635-651.

Farkas LM, Haffner C, Giger T, Khaitovich P, Nowick K, Birchmeier C, Paabo S, Huttner WB. 2008. Insulinoma-associated 1 has a panneurogenic role and promotes the generation and expansion of basal progenitors in the developing mouse neocortex. Neuron 60: 4055.

Goebbels S, Bormuth I, Bode U, Hermanson O, Schwab MH, Nave KA. 2006. Genetic targeting of principal neurons in neocortex and hippocampus of NEX-Cre mice. Genesis 44: 611621.

Gorski JA, Talley T, Qiu M, Puelles L, Rubenstein JL, Jones KR. 2002. Cortical excitatory neurons and glia, but not GABAergic neurons, are produced in the Emx1-expressing lineage. $J$ Neurosci 22: 6309-6314.

Grafodatskaya D, Chung B, Szatmari P, Weksberg R. 2010. Autism spectrum disorders and epigenetics. J Am Acad Child Adolesc Psychiatry 49: 794-809. 
Harnett D, Ambrozkiewicz MC, Zinnall U, Rusanova A, Borisova E, Dannenberg R, Imami K, Münster-Wandowski A, Fauler B, Mielke T et al. 2021. A critical period of translational control during brain development at codon resolution. bioRxiv: 2021.2006.2023.449626.

Heerma van Voss MR, Kammers K, Vesuna F, Brilliant J, Bergman Y, Tantravedi S, Wu X, Cole RN, Holland A, van Diest PJ et al. 2018. Global Effects of DDX3 Inhibition on Cell Cycle Regulation Identified by a Combined Phosphoproteomics and Single Cell Tracking Approach. Transl Oncol 11: 755-763.

Hoye ML, Silver DL. 2021. Decoding mixed messages in the developing cortex: translational regulation of neural progenitor fate. Curr Opin Neurobiol 66: 93-102.

Ingolia NT. 2016. Ribosome Footprint Profiling of Translation throughout the Genome. Cell 165: 22-33.

Iossifov I, O'Roak BJ, Sanders SJ, Ronemus M, Krumm N, Levy D, Stessman HA, Witherspoon KT, Vives L, Patterson KE et al. 2014. The contribution of de novo coding mutations to autism spectrum disorder. Nature 515: 216-221.

Jianghong $\mathrm{Ou} \mathrm{MLH}$. 2021. ribosomeProfilingQC: Ribosome Profiling Quality Control. . Bioconductor.

Jin X, Simmons SK, Guo A, Shetty AS, Ko M, Nguyen L, Jokhi V, Robinson E, Oyler P, Curry N et al. 2020. In vivo Perturb-Seq reveals neuronal and glial abnormalities associated with autism risk genes. Science $\mathbf{3 7 0}$.

Johnson-Kerner B, Snijders Blok L, Suit L, Thomas J, Kleefstra T, Sherr EH. 1993. DDX3XRelated Neurodevelopmental Disorder. in GeneReviews $((R))$ (eds. MP Adam, HH Ardinger, RA Pagon, SE Wallace, LJH Bean, G Mirzaa, A Amemiya), Seattle (WA).

Johnstone TG, Bazzini AA, Giraldez AJ. 2016. Upstream ORFs are prevalent translational repressors in vertebrates. EMBO J 35: 706-723.

Jones DT, Jager N, Kool M, Zichner T, Hutter B, Sultan M, Cho YJ, Pugh TJ, Hovestadt V, Stutz AM et al. 2012. Dissecting the genomic complexity underlying medulloblastoma. Nature 488: 100-105.

Kanai Y, Dohmae N, Hirokawa N. 2004. Kinesin transports RNA: isolation and characterization of an RNA-transporting granule. Neuron 43: 513-525.

Kellaris G, Khan K, Baig SM, Tsai IC, Zamora FM, Ruggieri P, Natowicz MR, Katsanis N. 2018. A hypomorphic inherited pathogenic variant in DDX3X causes male intellectual disability with additional neurodevelopmental and neurodegenerative features. Hum Genomics 12: 11.

Kotov AA, Olenkina OM, Kibanov MV, Olenina LV. 2016. RNA helicase Belle (DDX3) is essential for male germline stem cell maintenance and division in Drosophila. Biochim Biophys Acta 1863: 1093-1105.

Kraushar ML, Thompson K, Wijeratne HR, Viljetic B, Sakers K, Marson JW, Kontoyiannis DL, Buyske S, Hart RP, Rasin MR. 2014. Temporally defined neocortical translation and polysome assembly are determined by the RNA-binding protein Hu antigen R. Proc Natl Acad Sci U S A 111: E3815-3824.

Kriegstein A, Alvarez-Buylla A. 2009. The glial nature of embryonic and adult neural stem cells. Annu Rev Neurosci 32: 149-184.

Lange C, Huttner WB, Calegari F. 2009. Cdk4/cyclinD1 overexpression in neural stem cells shortens G1, delays neurogenesis, and promotes the generation and expansion of basal progenitors. Cell Stem Cell 5: 320-331.

Lee Y, Katyal S, Downing SM, Zhao J, Russell HR, McKinnon PJ. 2012. Neurogenesis requires TopBP1 to prevent catastrophic replicative DNA damage in early progenitors. Nat Neurosci 15: 819-826.

Lennox AL, Hoye ML, Jiang R, Johnson-Kerner BL, Suit LA, Venkataramanan S, Sheehan CJ, Alsina FC, Fregeau B, Aldinger KA et al. 2020. Pathogenic DDX3X Mutations Impair RNA Metabolism and Neurogenesis during Fetal Cortical Development. Neuron. 
Li Q, Zhang P, Zhang C, Wang Y, Wan R, Yang Y, Guo X, Huo R, Lin M, Zhou Z et al. 2014. DDX3X regulates cell survival and cell cycle during mouse early embryonic development. J Biomed Res 28: 282-291.

Li Z, Tyler WA, Zeldich E, Santpere Baro G, Okamoto M, Gao T, Li M, Sestan N, Haydar TF. 2020. Transcriptional priming as a conserved mechanism of lineage diversification in the developing mouse and human neocortex. Sci Adv 6.

Lodato S, Arlotta P. 2015. Generating neuronal diversity in the mammalian cerebral cortex. Annu Rev Cell Dev Biol 31: 699-720.

Madisen L, Zwingman TA, Sunkin SM, Oh SW, Zariwala HA, Gu H, Ng LL, Palmiter RD, Hawrylycz MJ, Jones AR et al. 2010. A robust and high-throughput Cre reporting and characterization system for the whole mouse brain. Nat Neurosci 13: 133-140.

Mao H, Pilaz LJ, McMahon JJ, Golzio C, Wu D, Shi L, Katsanis N, Silver DL. 2015. Rbm8a haploinsufficiency disrupts embryonic cortical development resulting in microcephaly. $J$ Neurosci 35: 7003-7018.

Mitchell-Dick A, Chalem A, Pilaz LJ, Silver DL. 2019. Acute Lengthening of Progenitor Mitosis Influences Progeny Fate during Cortical Development in vivo. Dev Neurosci 41: 300-317.

Monaghan CE, Nechiporuk T, Jeng S, McWeeney SK, Wang J, Rosenfeld MG, Mandel G. 2017. REST corepressors RCOR1 and RCOR2 and the repressor INSM1 regulate the proliferation-differentiation balance in the developing brain. Proc Natl Acad Sci U S A 114: E406-E415.

Nicola P, Blackburn PR, Rasmussen KJ, Bertsch NL, Klee EW, Hasadsri L, Pichurin PN, Rankin J, Raymond FL, Study DDD et al. 2019. De novo DDX3X missense variants in males appear viable and contribute to syndromic intellectual disability. Am J Med Genet A 179: 570-578.

Oh S, Flynn RA, Floor SN, Purzner J, Martin L, Do BT, Schubert S, Vaka D, Morrissy S, Li Y et al. 2016. Medulloblastoma-associated DDX3 variant selectively alters the translational response to stress. Oncotarget 7: 28169-28182.

Patmore DM, Jassim A, Nathan E, Gilbertson RJ, Tahan D, Hoffmann N, Tong Y, Smith KS, Kanneganti TD, Suzuki H et al. 2020. DDX3X Suppresses the Susceptibility of Hindbrain Lineages to Medulloblastoma. Dev Cell 54: 455-470 e455.

Pilaz LJ, McMahon JJ, Miller EE, Lennox AL, Suzuki A, Salmon E, Silver DL. 2016. Prolonged Mitosis of Neural Progenitors Alters Cell Fate in the Developing Brain. Neuron 89: 83-99.

Pilaz LJ, Patti D, Marcy G, Ollier E, Pfister S, Douglas RJ, Betizeau M, Gautier E, Cortay V, Doerflinger $\mathrm{N}$ et al. 2009. Forced G1-phase reduction alters mode of division, neuron number, and laminar phenotype in the cerebral cortex. Proc Natl Acad Sci U S A 106: 21924-21929.

Polioudakis D, de la Torre-Ubieta L, Langerman J, Elkins AG, Shi X, Stein JL, Vuong CK, Nichterwitz S, Gevorgian M, Opland CK et al. 2019. A Single-Cell Transcriptomic Atlas of Human Neocortical Development during Mid-gestation. Neuron 103: 785-801 e788.

Pugh TJ, Weeraratne SD, Archer TC, Krummel DAP, Auclair D, Bochicchio J, Carneiro MO, Carter SL, Cibulskis K, Erlich RL et al. 2012. Medulloblastoma exome sequencing uncovers subtype-specific somatic mutations. Nature 488: 106-110.

Quinn JC, Molinek M, Martynoga BS, Zaki PA, Faedo A, Bulfone A, Hevner RF, West JD, Price DJ. 2007. Pax6 controls cerebral cortical cell number by regulating exit from the cell cycle and specifies cortical cell identity by a cell autonomous mechanism. Dev Biol 302: 50-65.

RK CY, Merico D, Bookman M, J LH, Thiruvahindrapuram B, Patel RV, Whitney J, Deflaux N, Bingham J, Wang Z et al. 2017. Whole genome sequencing resource identifies 18 new candidate genes for autism spectrum disorder. Nat Neurosci 20: 602-611.

Robinson G, Parker M, Kranenburg TA, Lu C, Chen X, Ding L, Phoenix TN, Hedlund E, Wei L, Zhu XY et al. 2012. Novel mutations target distinct subgroups of medulloblastoma. Nature 488: $43-48$. 
Ruzzo EK, Perez-Cano L, Jung JY, Wang LK, Kashef-Haghighi D, Hartl C, Singh C, Xu J, Hoekstra JN, Leventhal O et al. 2019. Inherited and De Novo Genetic Risk for Autism Impacts Shared Networks. Cell 178: 850-866 e826.

Scala M, Torella A, Severino M, Morana G, Castello R, Accogli A, Verrico A, Vari MS, Cappuccio $\mathrm{G}$, Pinelli $\mathrm{M}$ et al. 2019. Three de novo DDX3X variants associated with distinctive brain developmental abnormalities and brain tumor in intellectually disabled females. Eur J Hum Genet 27: 1254-1259.

Silver DL RP, Grove EA, Haydar TF, Hensch TK, Huttner WB, Molnár Z, Rubenstein JL, Sestan N, Stryker MP, Sur M, Tosches MA, Walsh CA. 2019. Evolution and Ontogenetic Development of Cortical Structures. in The Neocortex (ed. ST Singer W, Rakic P), pp. 61109. MIT Press, Cambridge, MA.

Snijders Blok L, Madsen E, Juusola J, Gilissen C, Baralle D, Reijnders MR, Venselaar H, Helsmoortel C, Cho MT, Hoischen A et al. 2015. Mutations in DDX3X Are a Common Cause of Unexplained Intellectual Disability with Gender-Specific Effects on Wnt Signaling. Am J Hum Genet 97: 343-352.

Szappanos D, Tschismarov R, Perlot T, Westermayer S, Fischer K, Platanitis E, Kallinger F, Novatchkova M, Lassnig C, Muller M et al. 2018. The RNA helicase DDX3X is an essential mediator of innate antimicrobial immunity. PLoS Pathog 14: e1007397.

Takahashi T, Nowakowski RS, Caviness VS, Jr. 1995. The cell cycle of the pseudostratified ventricular epithelium of the embryonic murine cerebral wall. J Neurosci 15: 6046-6057.

Takata A, Miyake N, Tsurusaki Y, Fukai R, Miyatake S, Koshimizu E, Kushima I, Okada T, Morikawa M, Uno $Y$ et al. 2018. Integrative Analyses of De Novo Mutations Provide Deeper Biological Insights into Autism Spectrum Disorder. Cell Rep 22: 734-747.

Tang L, Levy T, Guillory S, Halpern D, Zweifach J, Giserman-Kiss I, Foss-Feig JH, Frank Y, Lozano R, Belani $P$ et al. 2021. Prospective and detailed behavioral phenotyping in DDX3X syndrome. Mol Autism 12: 36.

Telley L, Agirman G, Prados J, Amberg N, Fievre S, Oberst P, Bartolini G, Vitali I, Cadilhac C, Hippenmeyer $S$ et al. 2019. Temporal patterning of apical progenitors and their daughter neurons in the developing neocortex. Science 364.

Tsanov N, Samacoits A, Chouaib R, Traboulsi AM, Gostan T, Weber C, Zimmer C, Zibara K, Walter T, Peter M et al. 2016. smiFISH and FISH-quant - a flexible single RNA detection approach with super-resolution capability. Nucleic Acids Res 44: e165.

Tukiainen T, Villani AC, Yen A, Rivas MA, Marshall JL, Satija R, Aguirre M, Gauthier L, Fleharty $\mathrm{M}$, Kirby $A$ et al. 2017. Landscape of $X$ chromosome inactivation across human tissues. Nature 550: 244-248.

Venkataramanan S, Gadek M, Calviello L, Wilkins K, Floor SN. 2021. DDX3X and DDX3Y are redundant in protein synthesis. RNA 27: 1577-1588.

Wang X, Posey JE, Rosenfeld JA, Bacino CA, Scaglia F, Immken L, Harris JM, Hickey SE, Mosher TM, Slavotinek A et al. 2018. Phenotypic expansion in DDX3X - a common cause of intellectual disability in females. Ann Clin Transl Neurol 5: 1277-1285.

Wang Y, Wu Q, Yang P, Wang C, Liu J, Ding W, Liu W, Bai Y, Yang Y, Wang H et al. 2016. LSD1 co-repressor Rcor2 orchestrates neurogenesis in the developing mouse brain. Nat Commun 7: 10481.

Willsey AJ, Sanders SJ, Li M, Dong S, Tebbenkamp AT, Muhle RA, Reilly SK, Lin L, Fertuzinhos S, Miller JA et al. 2013. Coexpression networks implicate human midfetal deep cortical projection neurons in the pathogenesis of autism. Cell 155: 997-1007.

Willsey HR, Exner CRT, Xu Y, Everitt A, Sun N, Wang B, Dea J, Schmunk G, Zaltsman Y, Teerikorpi $\mathrm{N}$ et al. 2021. Parallel in vivo analysis of large-effect autism genes implicates cortical neurogenesis and estrogen in risk and resilience. Neuron 109: 788-804 e788.

Witecka A, Kwiatkowski S, Ishikawa T, Drozak J. 2021. The Structure, Activity, and Function of the SETD3 Protein Histidine Methyltransferase. Life (Basel) 11. 
Wu H, Luo J, Yu H, Rattner A, Mo A, Wang Y, Smallwood PM, Erlanger B, Wheelan SJ, Nathans J. 2014. Cellular resolution maps of $X$ chromosome inactivation: implications for neural development, function, and disease. Neuron 81: 103-119.

Yang G, Smibert CA, Kaplan DR, Miller FD. 2014. An elF4E1/4E-T complex determines the genesis of neurons from precursors by translationally repressing a proneurogenic transcription program. Neuron 84: 723-739.

Zahr SK, Yang G, Kazan H, Borrett MJ, Yuzwa SA, Voronova A, Kaplan DR, Miller FD. 2018. A Translational Repression Complex in Developing Mammalian Neural Stem Cells that Regulates Neuronal Specification. Neuron 97: 520-537 e526.

Zhang L, Li X. 2021. DEAD-Box RNA Helicases in Cell Cycle Control and Clinical Therapy. Cells 10. 
Figure 1

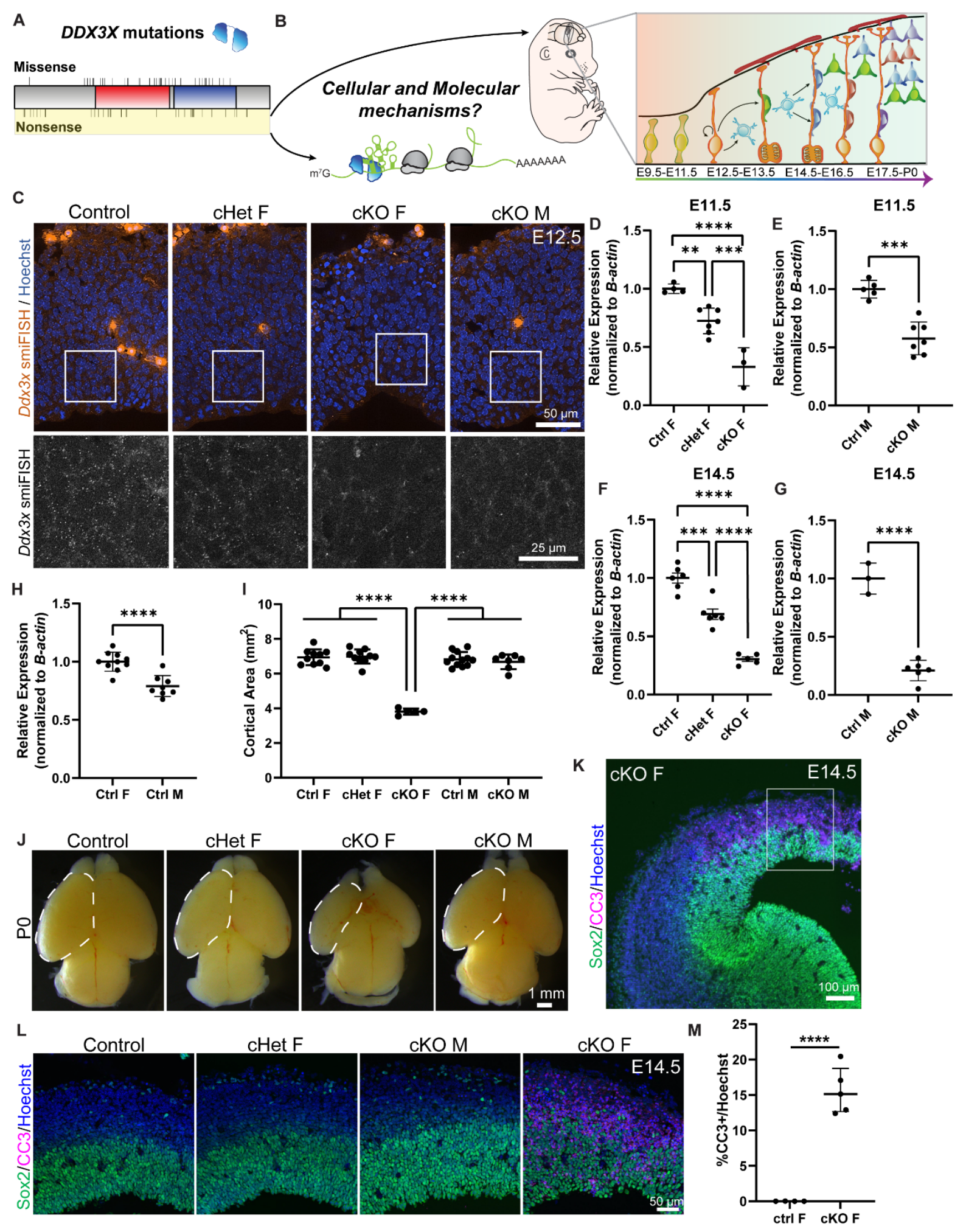


Figure 1. Conditional knockout of $D d x 3 x$ in neural progenitors using Emx1-Cre leads to microcephaly in female mice. $(A)$ Schematic of DDX3X protein with human missense and nonsense mutations noted, along with helicase/RNA binding domains (red, blue). Nonsense mutations, highlighted in yellow, are predicted to act in a LoF manner. (B) (Left) DDX3X protein bound to an mRNA undergoing translation. (Right) Mouse embryo and corticogenesis showing neuroepithelial cells (light green), radial glial cells (RGCs, orange), intermediate progenitors (IPs, light blue), and neurons (multi-colored). This study asks how does $D d x 3 x$ LoF impair mouse embryonic cortical development at a cellular and molecular level? (C) Representative sections of smiFISH for $D d x 3 x$ in control, cHet female, and cKO male and female E12.5 cortices. (D-G) Validation of $D d \times 3 \times$ mRNA knockdown in Tdtomato+ cells from control, cHet, and cKO female $(D$, $F)$ and control and cKO male $(E, G)$ brains sorted via FACS at E11.5 $(D, E)$ and $E 14.5(F, G) .(H)$ Quantification of $D d \times 3 x$ levels in Tdtomato+ cells from control female and male brains. $(I)$ Quantification of cortical area at P0. (J) Representative whole mount images of control, cHet female, and cKO male and female brains at P0. $(K, L)$ Representative sections of E14.5 brains stained with Sox2 (green), CC3 (magenta) and Hoechst (blue). (M) Quantification of CC3+ cells in E14.5 control and cKO female cortices. Scale bars, indicated. Error bars, S.D. ${ }^{* *} p<0.01$, ${ }^{* * *} p<0.001,{ }^{* * *} p<0.0001$. 
Figure 2

A

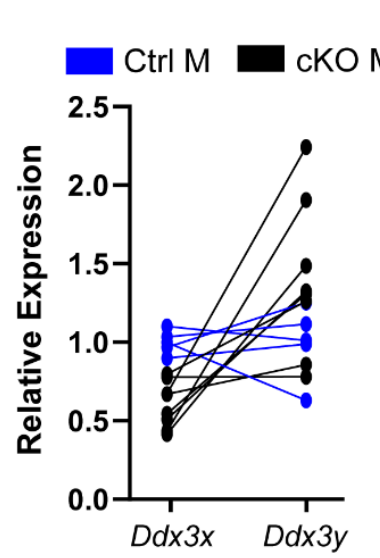

B
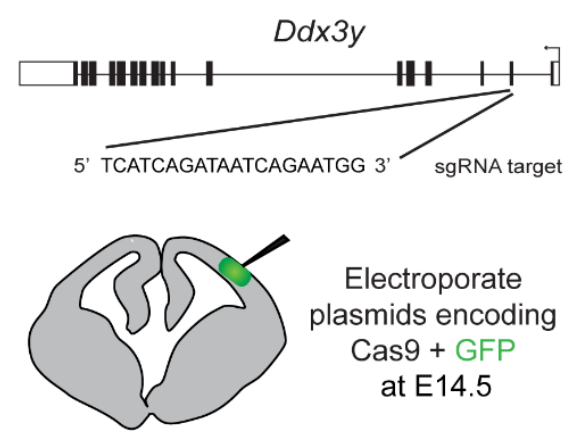

Electroporate plasmids encoding Cas9 + GFP at E14.5
C

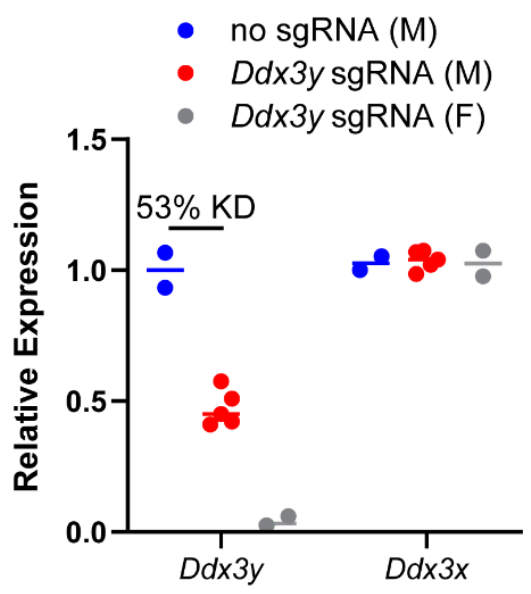

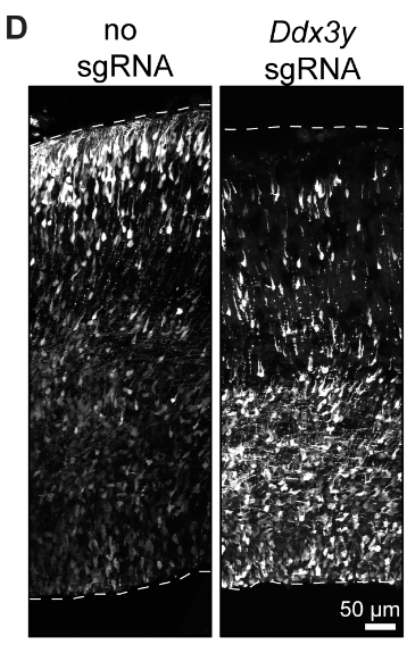

$\mathrm{E} 14.5 \rightarrow \mathrm{E} 17.5$

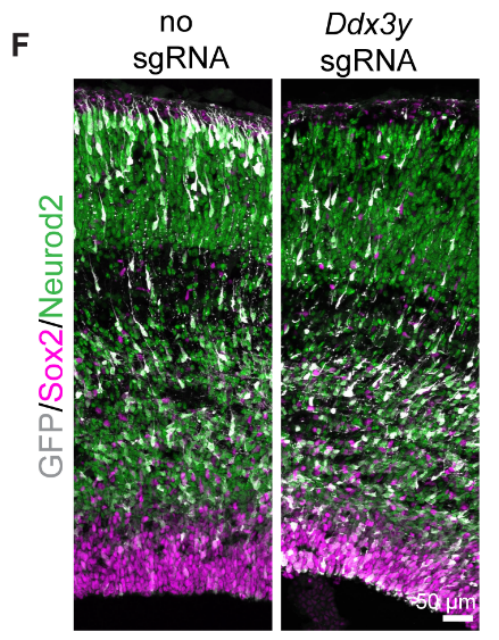

$\mathrm{E} 14.5 \rightarrow \mathrm{E} 17.5$

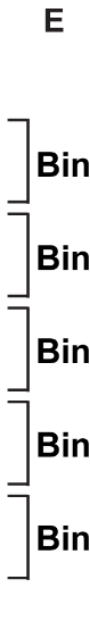

- no sgRNA (males)

- Ddx3y sgRNA (males)

Daxjy sgRna (i

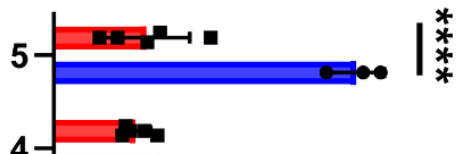

\section{$\stackrel{*}{*}$}

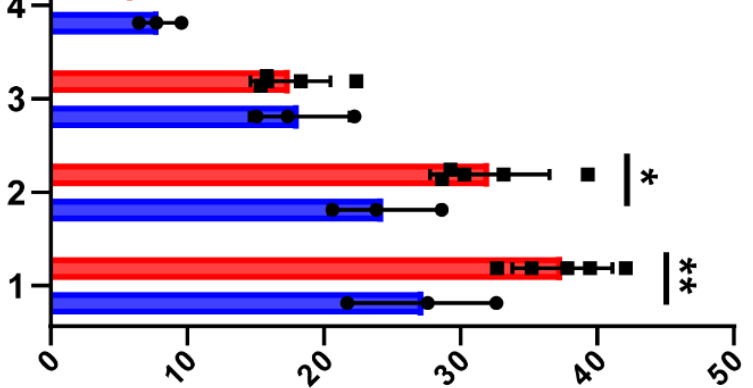

Percentage of GFP+ cells

G

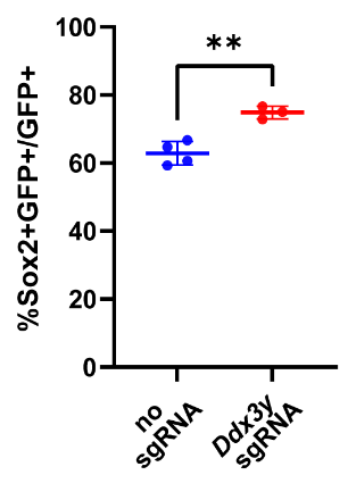

H

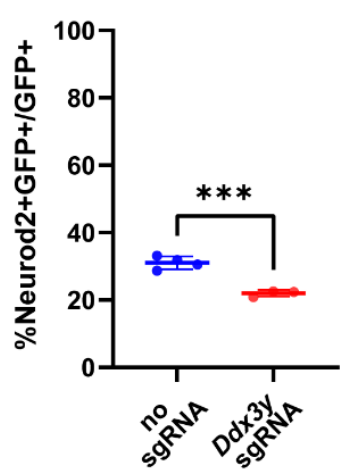


Figure 2. $D d x 3 x$ knockout is sexually dimorphic and $D d x 3 y$ phenocopies $D d x 3 x$ loss. $(A)$ RT-qPCR quantification of $D d x 3 x$ and $D d x 3 y$ mRNA levels in FACS-isolated Tdtomato+ cells from cKO male E11.5 cortices. (B) Schematic of Ddx3y CRISPR sgRNA electroporation of E14.5 brain. (C) RT-qPCR quantification of $D d x 3 y$ and $D d x 3 x$ levels in GFP+ FACS-isolated cells from E17.5 male and female mice electroporated with pCAG-GFP and either no sgRNA or $D d x 3 y$ sgRNA. $(D)$ Representative sections of E17.5 male brains electroporated at E14.5 with pCAG-GFP and either no sgRNA or Ddx3y sgRNA and stained with anti-GFP (grey). Dotted lines, ventricular and pial surfaces; brackets delineate equivalently sized bins. $(E)$ Quantification of distribution of GFP+ cells. $(F)$ Same as $(D)$, but sections were stained with anti-GFP (grey), Sox2 (magenta), and Neurod2 (red). $(G, H)$ Quantification of GFP co-localization with Sox2 $(G)$ or Neurod2 $(H)$. Scale bars, indicated. Error bars, S.D. ${ }^{* *} p<0.01,{ }^{* *} p<0.001$. 
Figure 3
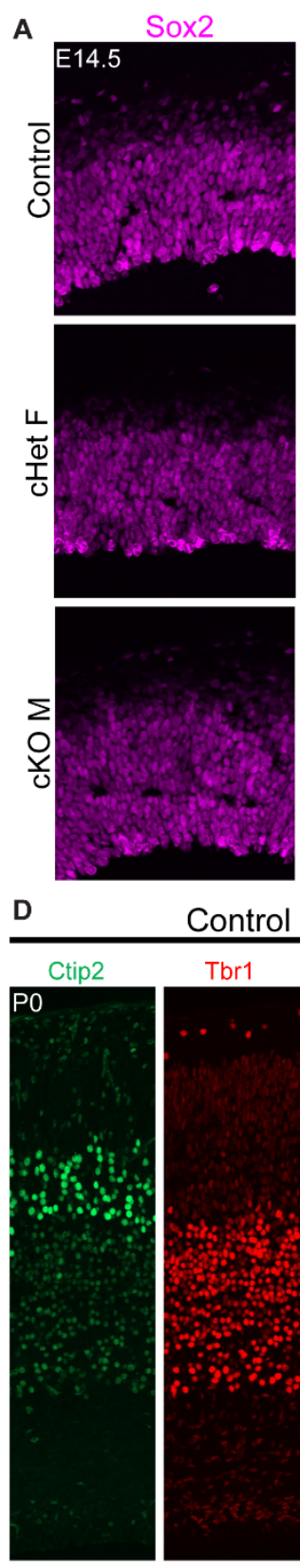

E

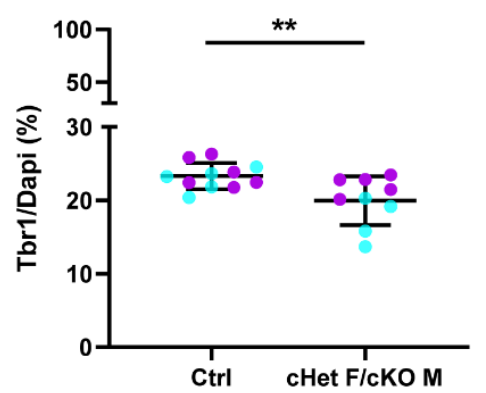

Tbr2

Tbr1
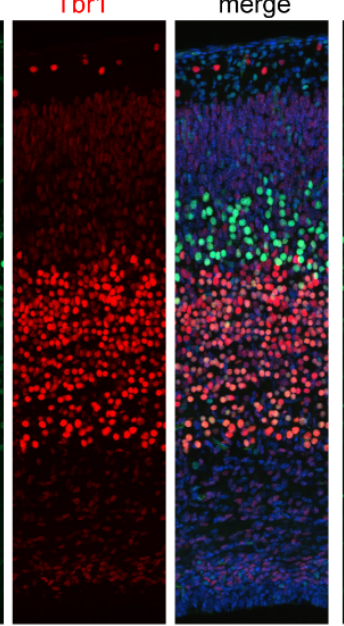

Tbr1 Density
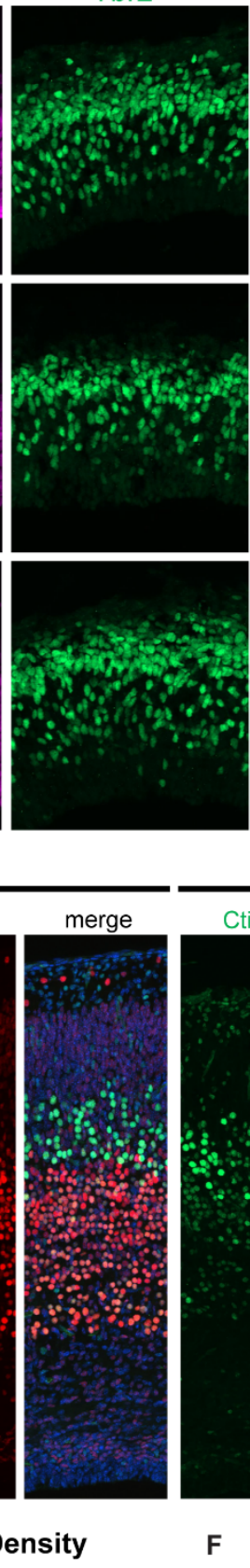

F

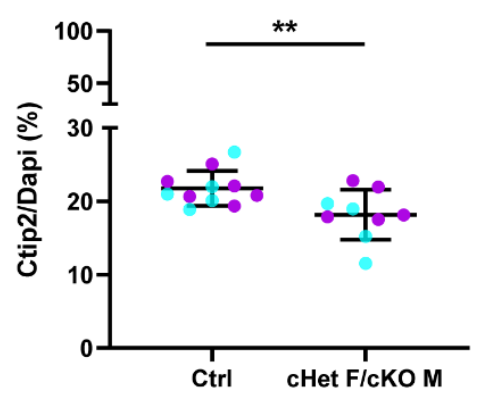

merge
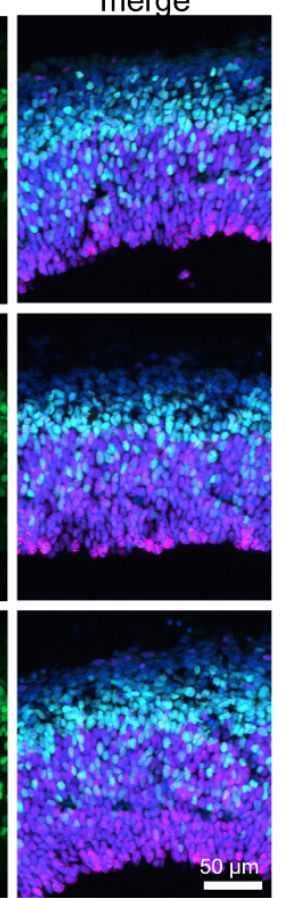

cHet F/cKO M
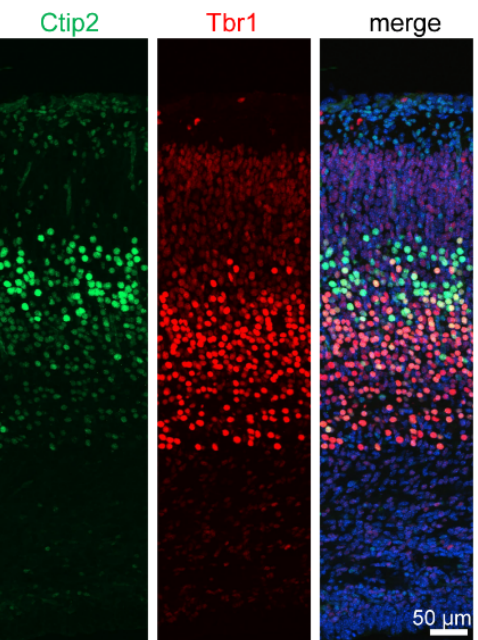

$50 \mu \mathrm{m}$
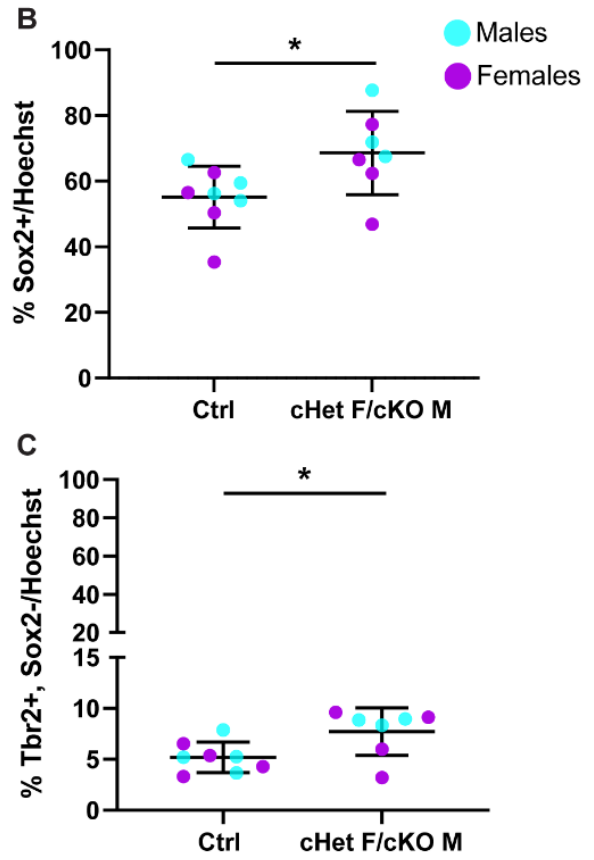

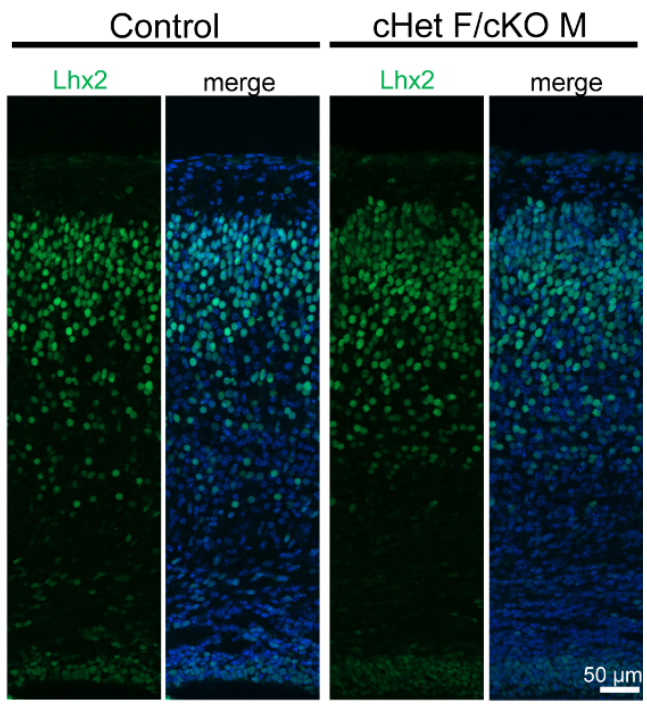

\section{G}

\section{Lhx2 Density}

Males
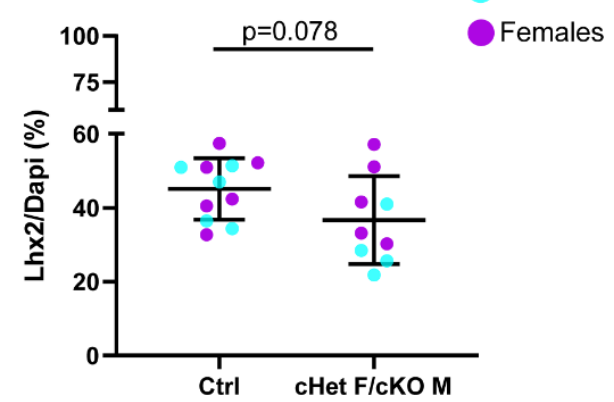
Figure 3. $D d x 3 x$ depletion leads to more RGCs and mature IPs, and fewer excitatory neurons across laminar layers. $(A)$ Representative sections from control, cHet female, and cKO male E14.5 cortices stained with Sox2 (magenta) and Tbr2 (green). (B, C) Quantification of density of Sox2+ $(B)$ and Tbr2+Sox2- $(C)$ cells relative to all cells (Hoechst) at E14.5. $(D)$ Representative sections stained with Ctip2 (green), Tbr1 (red), and Lhx2 (green) from P0 control and $\mathrm{cHet} F / \mathrm{CKO} \mathrm{M}$ cortices (control M and cKO M shown). (E-G) Quantification of laminar marker density for Tbr1 $(E)$, Ctip2 $(F)$, and Lhx2 $(G)$ relative to all cells (Hoechst). Scale bars, indicated. Error bars, S.D. ${ }^{*} p<0.05,{ }^{* *} p<0.01$. 


\section{Figure 4}

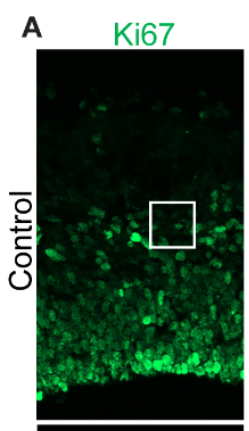

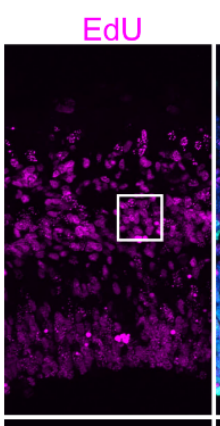

merge
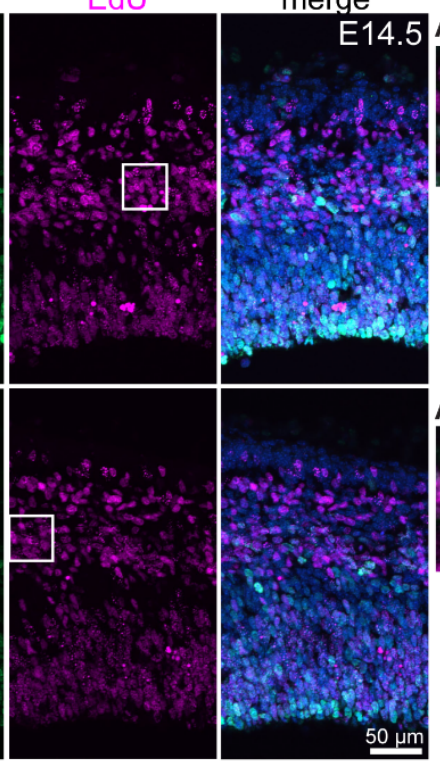

B

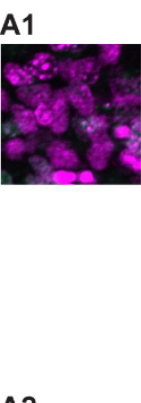

A2

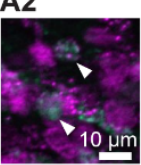

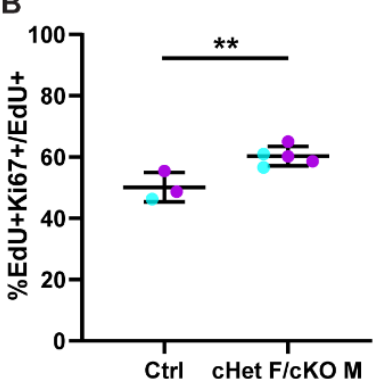
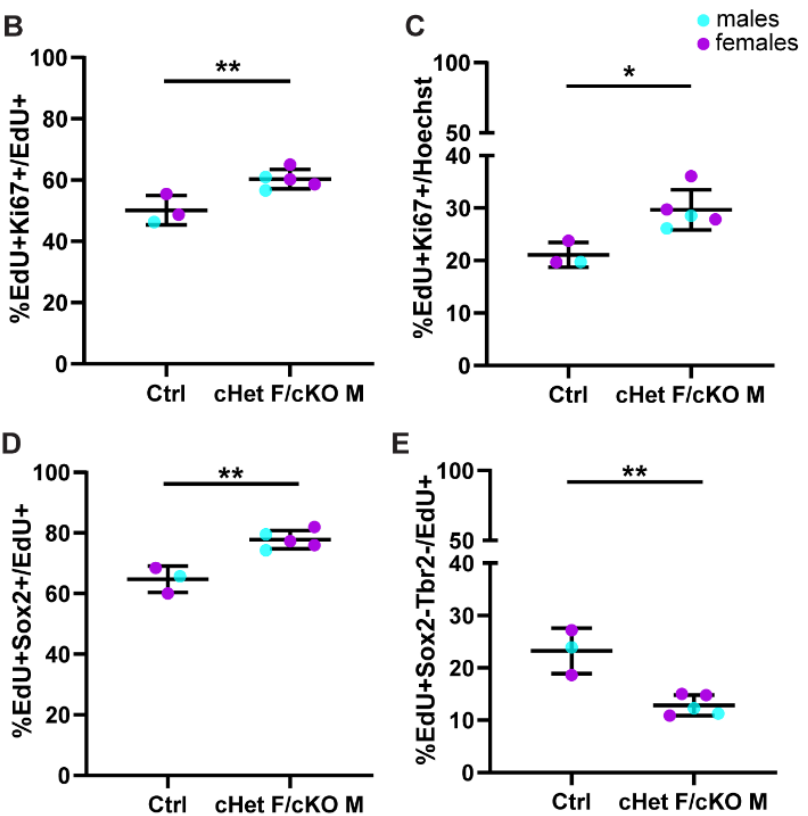

E

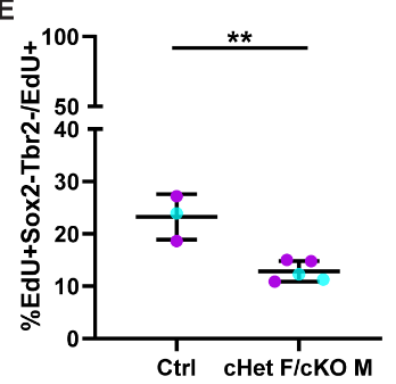

G

Ki67
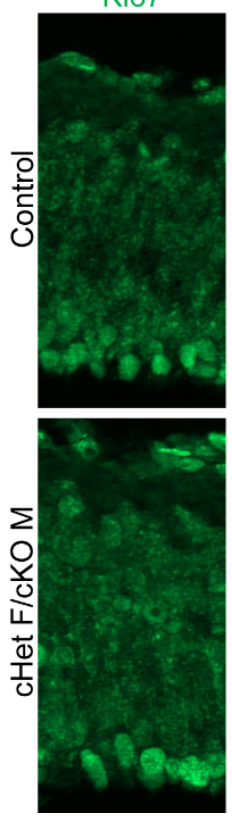

EdU
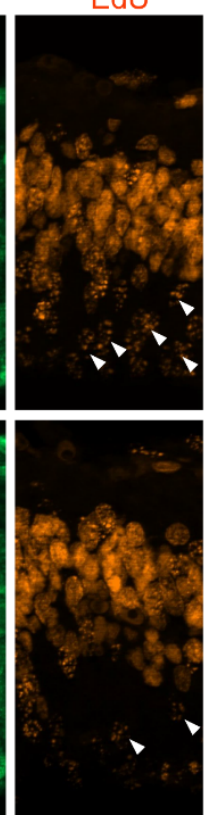

BrdU
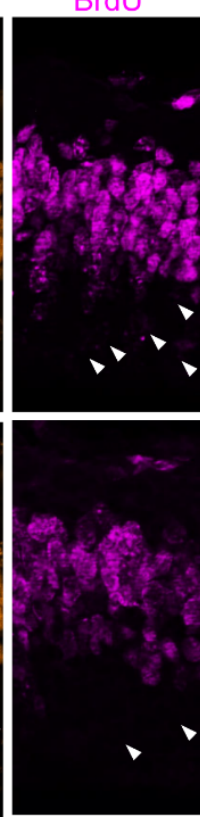

merge
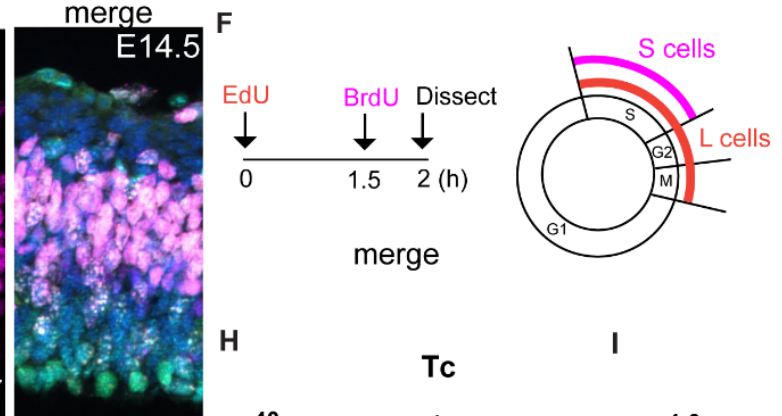

$\mathrm{T}_{\mathrm{s}}=\frac{\mathrm{S}_{\text {cells }}}{\mathrm{L}_{\text {cells }}} \times 1.5 \mathrm{hrs}$

$\mathrm{P}_{\text {cells }}=\mathrm{Ki67+}$

$T_{c}=\frac{P_{\text {cells }}}{S_{\text {cells }}} X T_{s}$

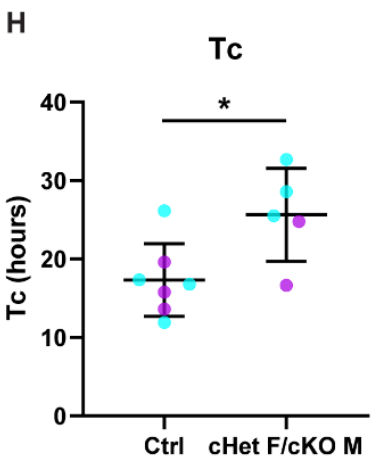

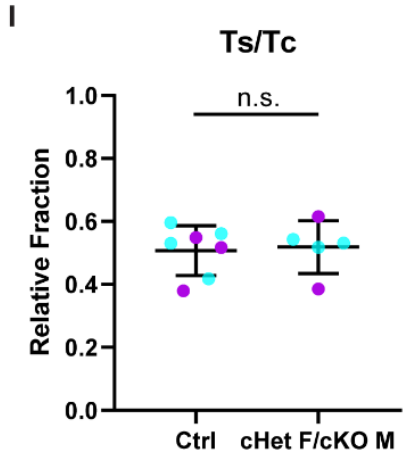


Figure 4. $D d x 3 x$ depletion impairs progenitor cell cycle exit and prolongs cell cycle duration. (A) Representative sections stained with Ki67 (green) and EdU (magenta) from E14.5 control and cHet F/CKO M mice (control F and cHet F shown) pulsed with EdU at E13.5, and higher magnification insets $(A 1, A 2) .(B, C)$ Quantification of Ki67+EdU+ relative to EdU+ cells $(B)$ and all cells (Hoechst, $C)$. (D, E) Quantification of EdU+Sox2+ $(D)$ and EdU+Sox2-Tbr2- cells $(E)$ relative to all EdU+ cells. $(F)$ Schematic illustrating the semi-cumulative labeling paradigm and cell cycle formulas. $(G)$ Representative sections of E14.5 control and cHet F/cKO M brains stained with Ki67 (green), EdU (red) and BrdU (magenta) and pulsed with EdU and BrdU (control M and cKO M shown). Arrows indicate EdU+BrdU- cells (ie: leaving cells). (H) Quantification of cell cycle duration (Tc) in control and cHet F/cKO M. (I) Quantification of Ts/Tc in control and cHet F/cKO M. Scale bars, indicated. Error bars, S.D. ${ }^{*} p<0.05,{ }^{* *} p<0.01$. 


\section{Figure 5}
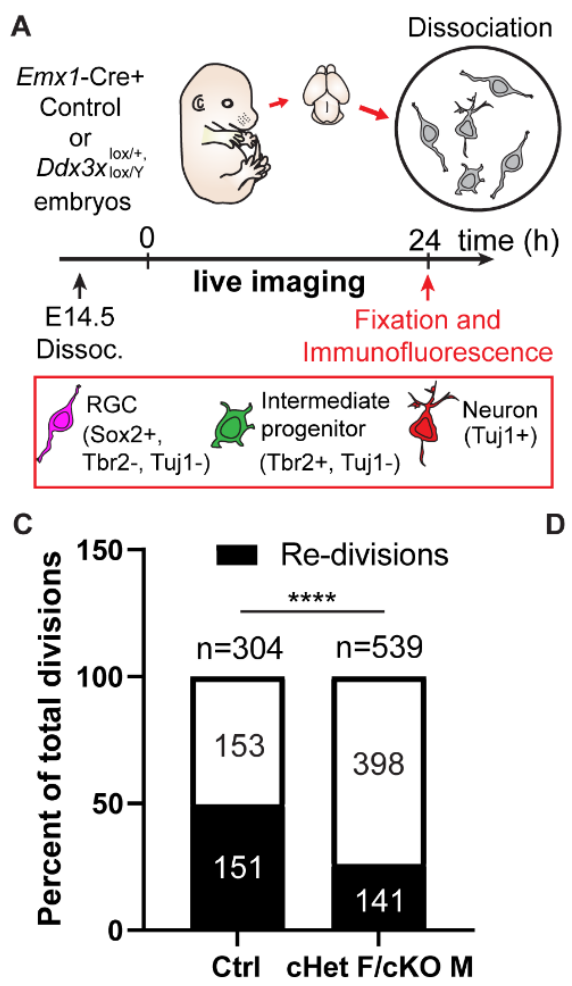

$\mathbf{B}$

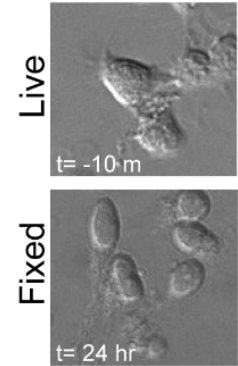

DIC

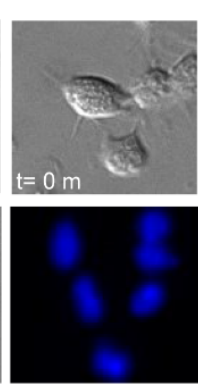

Hoechst

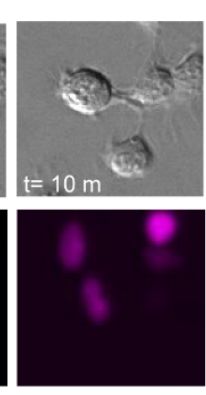

$\operatorname{Sox} 2$

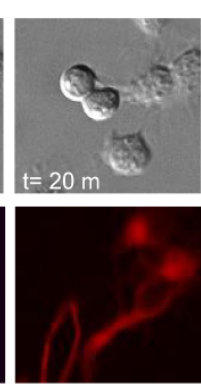

Tuj1

E

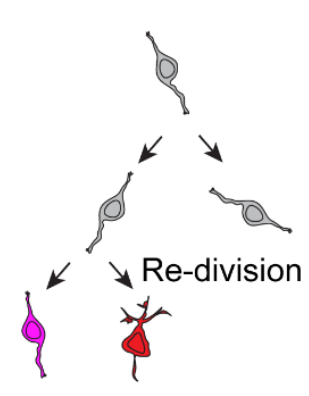

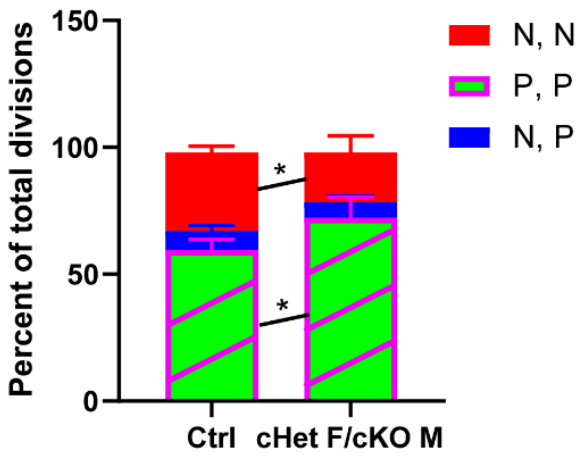

Figure 5. $D d \times 3 x$-depleted progenitors exhibit prolonged cell division and more proliferative divisions at the expense of neurogenic divisions. $(A)$ Live imaging paradigm for monitoring cell fate. $(B)$ Live imaging DIC snapshots at indicated $\mathrm{t}=$ minutes or hours, and fixed images stained with indicated markers. (C) Quantification of re-divisions in control and cHet F/cKO M. (D) Schematic illustrating an example of a re-division. $(E)$ Quantification of cell fate for P,P divisions (2 Sox2+ RGCs, or 2 Tbr2+ IPs, or 1 Sox2+ RGC and 1 Tbr2+Tuj1- IP); P, N divisions (1 Tuj1+ neuron and either 1 Sox2+ RGC or 1Tbr2+ IP); N, N divisions (2 Tuj1+ neurons). Scale bars, indicated. Error bars, S.D. ${ }^{*} p<0.05$. 
Figure 6
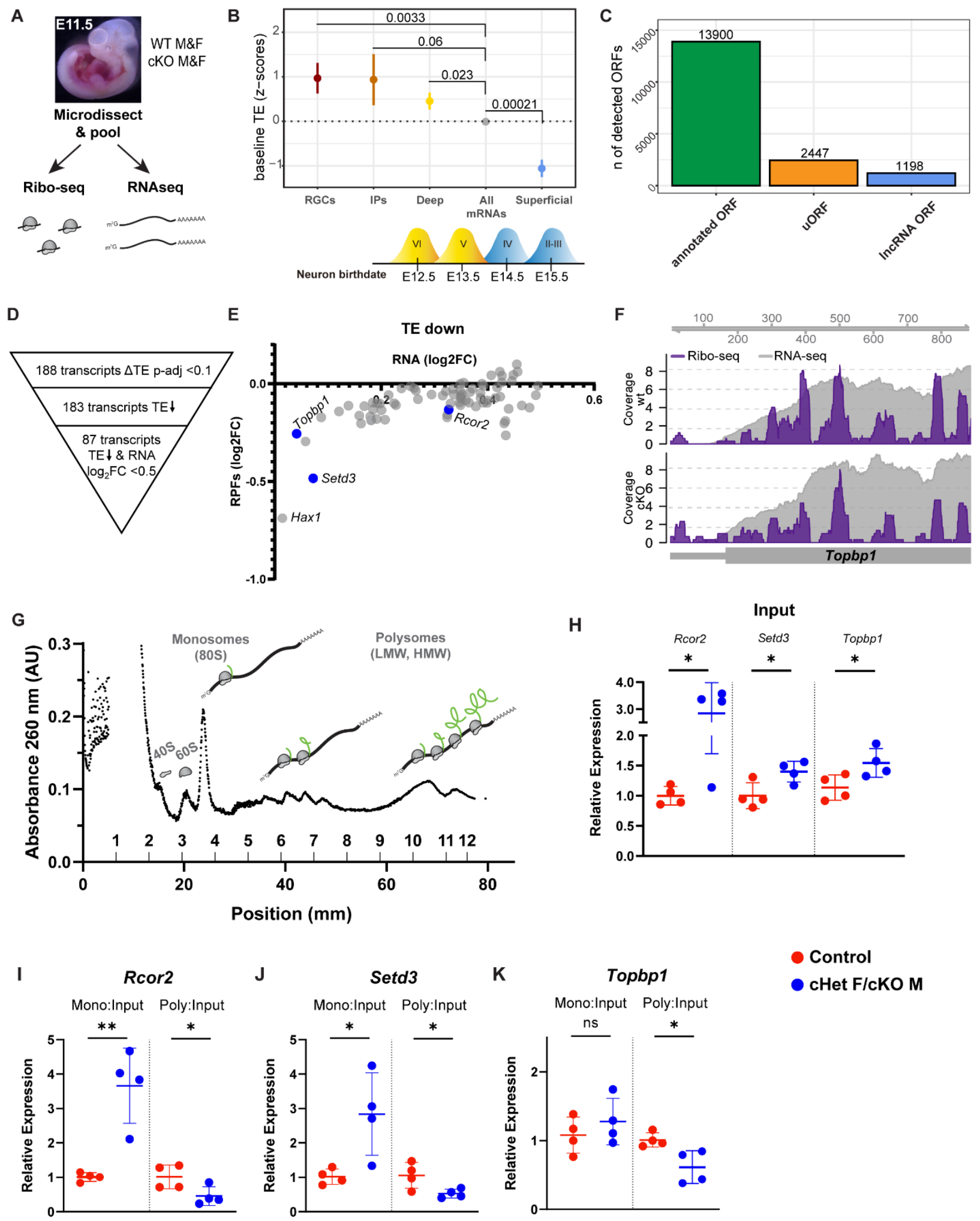

- Control

- cHet F/cKO M 
Figure 6. Ribosome Profiling in wild-type and Ddx3x cKO embryonic brains identifies DDX3X-dependent translation targets. $(A)$ Experimental paradigm for Ribo-seq and RNAseq of E11.5 cortices from control and cKO mice. $(B)$ TE of transcripts enriched in RGCs, IPs, deep layer neurons (VI-V) and superficial layer neurons (IV-II) relative to all other mRNAs (TPM >10). Birthdates for laminar layers are indicated below. See supplemental excel 3 for exact transcripts. (C) ORFquant analysis of wildtype Ribo-seq data showing identification of annotated ORFs and uORFs in protein-coding and non-coding isoforms. (D) Schematic illustrating how DDX3Xdependent targets were prioritized. $(E)$ Scatter plot of RPFs log2FC versus RNA log2FC for 87 DDX3X-dependent targets with significantly lower TE. Putative Ribo-seq targets selected for validation are highlighted in blue. (F) IGV screenshots illustrating RNAseq reads (gray) and RPFs (Ribo-seq; purple) for Topbp1 in cKO mice relative to control. (G) Representative trace from polysome fractionation. (H-K) RT-qPCR quantification of mRNA levels for Ribo-seq candidates in input sample $(H)$, monosome, and polysome fractions. Error bars, S.D. ${ }^{*} p<0.05,{ }^{* *} p<0.01$. 


\section{Figure 7}
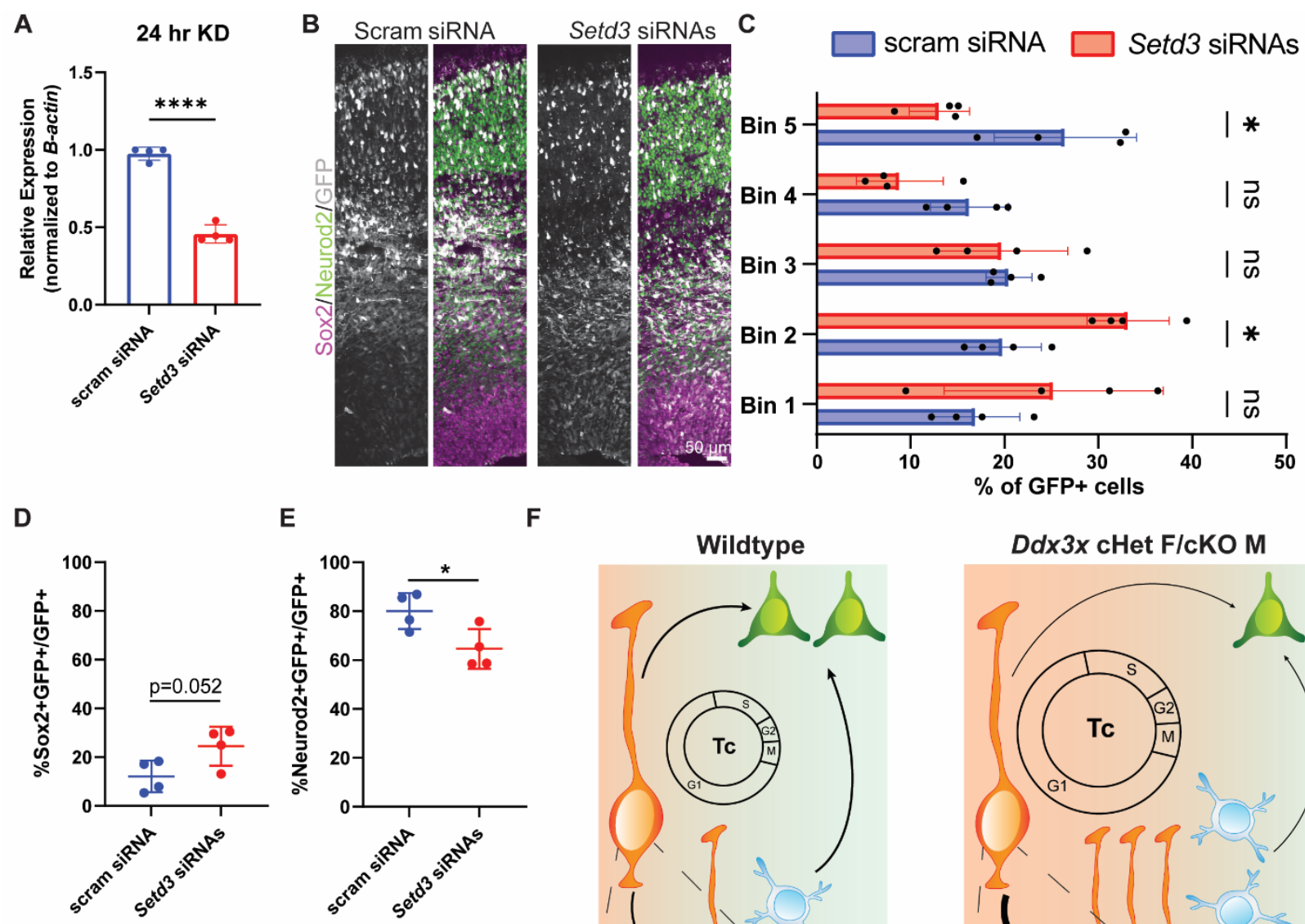

E

$\mathbf{F}$

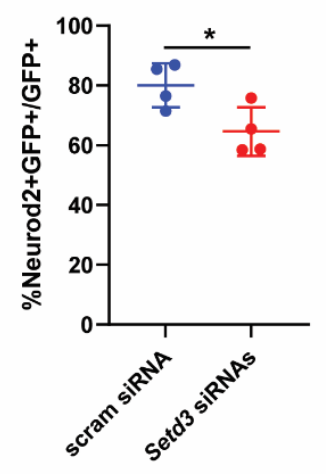

DDX3X-dependent neurogenic mRNA
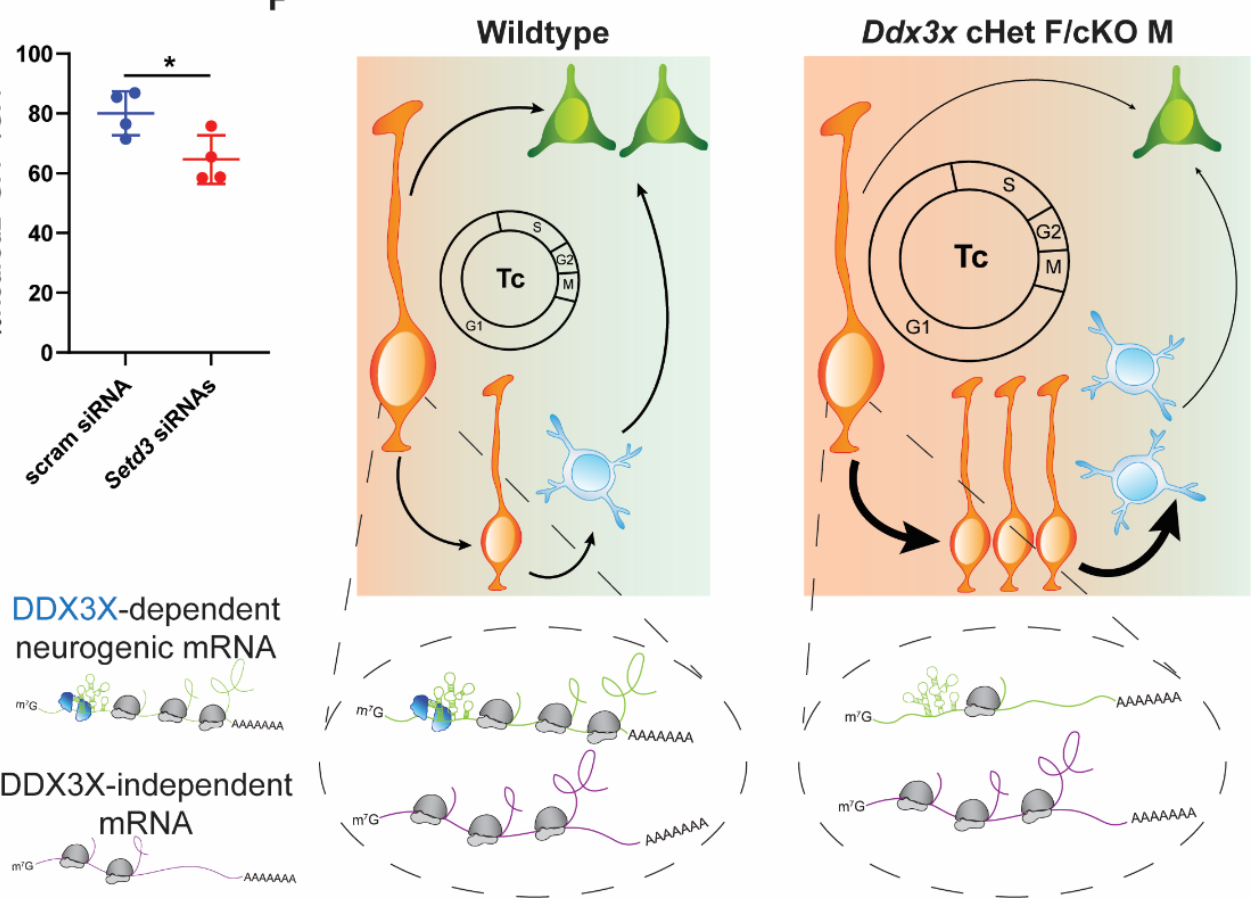
Figure 7. DDX3X-dependent translation target, Setd3, is required for neurogenesis. $(A)$ RTqPCR quantification of Setd3 knockdown in N2A cells. (B) Representative sections of E17.5 brains from mice electroporated at E14.5 with pCAG-GFP and scrambled or Setd3 siRNAs and immunostained with GFP (grey), Sox2 (magenta), Neurod2 (green). (C) Quantification of distribution of GFP-positive cells in 5 even bins of cortex. $(D, E)$ Quantification of GFP colocalization with Sox2+ $(D)$ and Neurod2+ $(E)$ cells. $(F)$ Schematic model summarizing how loss of DDX3X-dependent translation impairs neurogenesis. Scale bars, indicated. Error bars, S.D. ${ }^{*} p<0.05,{ }^{* * * *} p<0.0001$ 